\title{
Porous lanthanide metal-organic frameworks with metallic conductivity
}

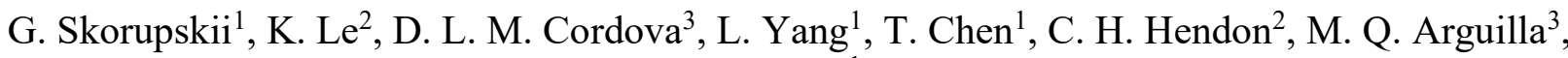 \\ M. Dincă ${ }^{1 *}$ \\ ${ }^{1}$ Department of Chemistry, Massachusetts Institute of Technology; Cambridge, \\ Massachusetts, United States. \\ ${ }^{2}$ Department of Chemistry, University of Oregon; Eugene, Oregon, United States. \\ ${ }^{3}$ Department of Chemistry, University of California, Irvine; Irvine, California, United States. \\ *Corresponding author. Email: mdinca@mit.edu
}

\begin{abstract}
Metallic charge transport and porosity appear almost mutually exclusive. Whereas metals demand large numbers of free carriers and must have minimal impurities and lattice vibrations to avoid charge scattering, the voids in porous materials limit the carrier concentration, provide ample space for impurities, and create more charge-scattering vibrations due to the size and flexibility of the lattice. No microporous material has been conclusively shown to behave as a metal. Here, we demonstrate that single crystals of the porous metal-organic framework $\operatorname{Ln}_{1.5}$ HOTP ( $\mathrm{Ln}=\mathrm{La}, \mathrm{Nd}$; HOTP $=2,3,6,7,10,11$-hexaoxytriphenylene) show the highest roomtemperature conductivities of all porous materials, along with clear temperature-deactivated transport. A structural transition consistent with charge density wave ordering, present only in metals and rare in any materials, provides additional conclusive proof of the metallic nature of the materials.
\end{abstract}

\section{Main Text:}

Microporous metals are not known. The defining characteristic of a metal is an inverse relationship between its electrical conductivity and temperature. This is due to scattering: the mobility of charge carriers is lowered when they interact with, or scatter off of impurities or lattice vibrations (i.e. phonons) - which themselves intensify with temperature (1). In porous materials, scattering processes are further intensified by the presence of physical voids, which also reduce the charge carrier concentration, as well as typically larger unit cells, which provide additional highamplitude vibrations, and higher concentrations of defects and impurities. Despite its seemingly antithetical nature, the combination of electrical conductivity and porosity is critical for a variety of applications, such as charge storage (2) or electrocatalysis (3). Materials that show both of these features are extremely rare, with activated carbons (4) or holey graphenes (5) overwhelmingly represented in the literature. These, however, are not metallic, and are not easily tunable chemically. One set of porous materials that provides a wide range of chemical tunability is metalorganic frameworks (MOFs). 
MOFs are generally insulating because they lack efficient pathways for charge transport. First, their lattices are generally ionic, meaning the linkages between the constituent organic ligands and the metal nodes create localized electronic states and impede charge delocalization. Second, the contents of the pores: solvent molecules, inert gas, or vacuum, are themselves insulating (6). Despite these challenges, several recent reports show that electrical conductivity can exist in certain types of MOFs, especially when the ligands enable strong charge delocalization (7-9). Frameworks built with triphenylene-based ligands stand out in this sense because the flat aromatic ligands form strongly delocalized orbitals with the metal ions, and have a strong propensity for $\pi$ $\pi$ stacking, combining the defining features of traditional inorganic and organic conductors $(7,8$, 10). Indeed, porous triphenylene-based MOFs have shown conductivities on the level of $100 \mathrm{~S} / \mathrm{cm}$ (11), comparable to graphite. However, even though some of these materials are computationally predicted to be metallic, there is only circumstantial evidence confirming their metallic nature. At best, this behavior coexists with and is overwhelmed by much clearer signs of semiconductor behavior $(7,12-15)$.

Here, we report that high-quality single crystals of $\operatorname{Ln}_{1.5}(2,3,6,7,10,11$-hexaoxytriphenylene) (Ln1.5HOTP; $\operatorname{Ln}=\mathrm{La}, \mathrm{Nd}$ ) demonstrate clear, temperature-deactivated - metallic - conductivity. They further demonstrate the highest room-temperature electrical conductivity among all MOFs or any other intrinsically porous material, reaching values above $1000 \mathrm{~S} / \mathrm{cm}$. As a unique, and rare signature of one-dimensional metals, the materials also undergo an incommensurate ordering transition consistent with the formation of a charge density wave (CDW) state. The latter is possible only in metallic materials and has never been observed for a MOF or any other porous material. The CDW transition temperature, $360 \mathrm{~K}-375 \mathrm{~K}$, is the highest for any organic-based material. The MOFs crystallize under easily accessible synthetic conditions from commercially available precursors, thus providing a convenient and tunable platform for studying the complex physics arising from the combination of the metallic charge transport and porosity.

Under conditions mimicking those reported previously for the cation-deficient isostructural analogs Ln $\mathbf{L n}_{1.5-x}$ HOTP $(x=0.2-0.3)(10), \mathrm{H}_{6} \mathrm{HOTP}$ reacts with a large excess of $\mathrm{Ln}\left(\mathrm{NO}_{3}\right)_{3}$ to produce dark blue/purple, highly reflective, needle-shaped crystals of Ln 1.5 HOTP (Fig. 1E). Single-crystal X-ray diffraction data collected above $375 \mathrm{~K}$ show that both frameworks crystallize in monoclinic systems (space group P2 $1 / 1$ for La1.5HOTP and C2/c for Nd1.5HOTP). A portion of the crystal structure of La1.5HOTP at $375 \mathrm{~K}$ (Fig. 1), essentially identical to that of Nd 1.5HOTP, shows columns of closely stacked HOTP linkers connected into a honeycomb net by eightcoordinate square-antiprismatic lanthanide ions. The apparent hexagonal symmetry of both structures is broken by only $10 \%$ of the metal sites, which deviate from their idealized position and lead to heavy twinning. 

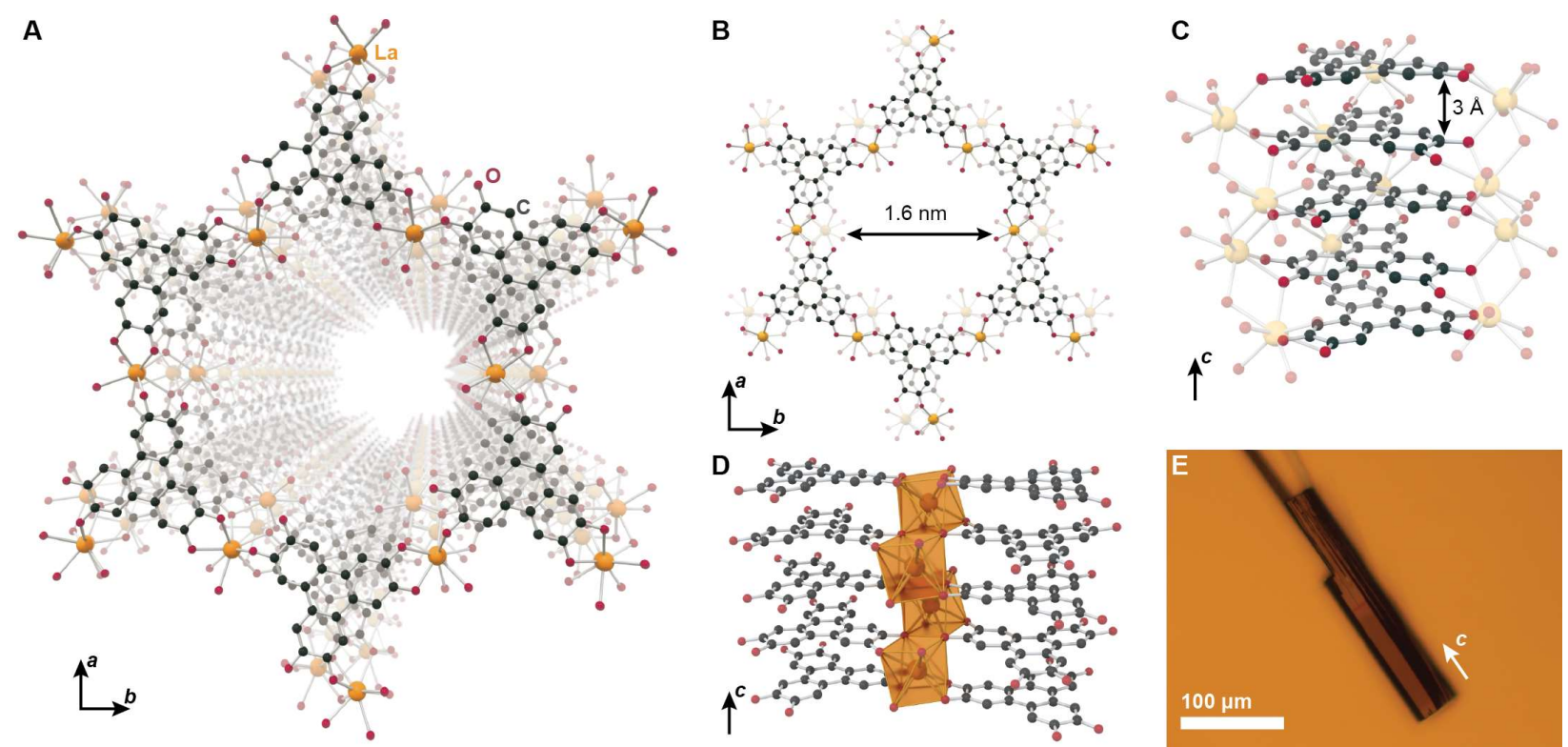

Fig. 1. Crystal structure of La ${ }_{1.5}$ HOTP. (A-D) The structure features large accessible one-dimensional channels (A), formed inside honeycomb-like layers (B) of the trigonal HOTP linkers connected by continuous $-\mathrm{La}-\mathrm{O}-$ chains, with the $\mathrm{La}^{3+}$ ions in square antiprismatic environment (D). The structure features extensive $\pi-\pi$ stacking interactions between the linkers, which are separated by only $3.0 \AA$ at room temperature (C). (E) The material forms dark blue or purple hexagonal needles, with the crystallographic $\mathrm{c}$ direction, which is collinear to $\pi-\pi$ stacking in the material, aligned along the longer dimension of the crystals. In the models, orange shows metal atoms, red oxygen, and dark grey carbon. Hydrogen atoms, minority disorder components, and uncoordinated solvent molecules were omitted for clarity.

Computed electronic band structures of Ln1.5HOTP, obtained by density functional theory (DFT) calculations (Fig. 2A-B), show that the materials possess electronic bands that resemble onedimensional metals, with disperse bands crossing the Fermi level along the $\mathrm{A}-\Gamma$ vector, and relatively flat bands along other vectors, consistent with data previously found for $\mathbf{L n}_{1.5-x}$ HOTP (10). La1.5HOTP and Nd1.5HOTP are qualitatively similar electronically, except for additional bands brought by the partially filled $4 f$ orbitals of $\mathrm{Nd}^{3+}$. The strong electronic anisotropy evidenced in the band diagrams is justified by considering the covalent and non-covalent interactions within the material: the Ln-O bonds that provide connectivity within the honeycomb layers are highly ionic (16), allow only negligible charge delocalization, and thus give rise to flat bands along the 

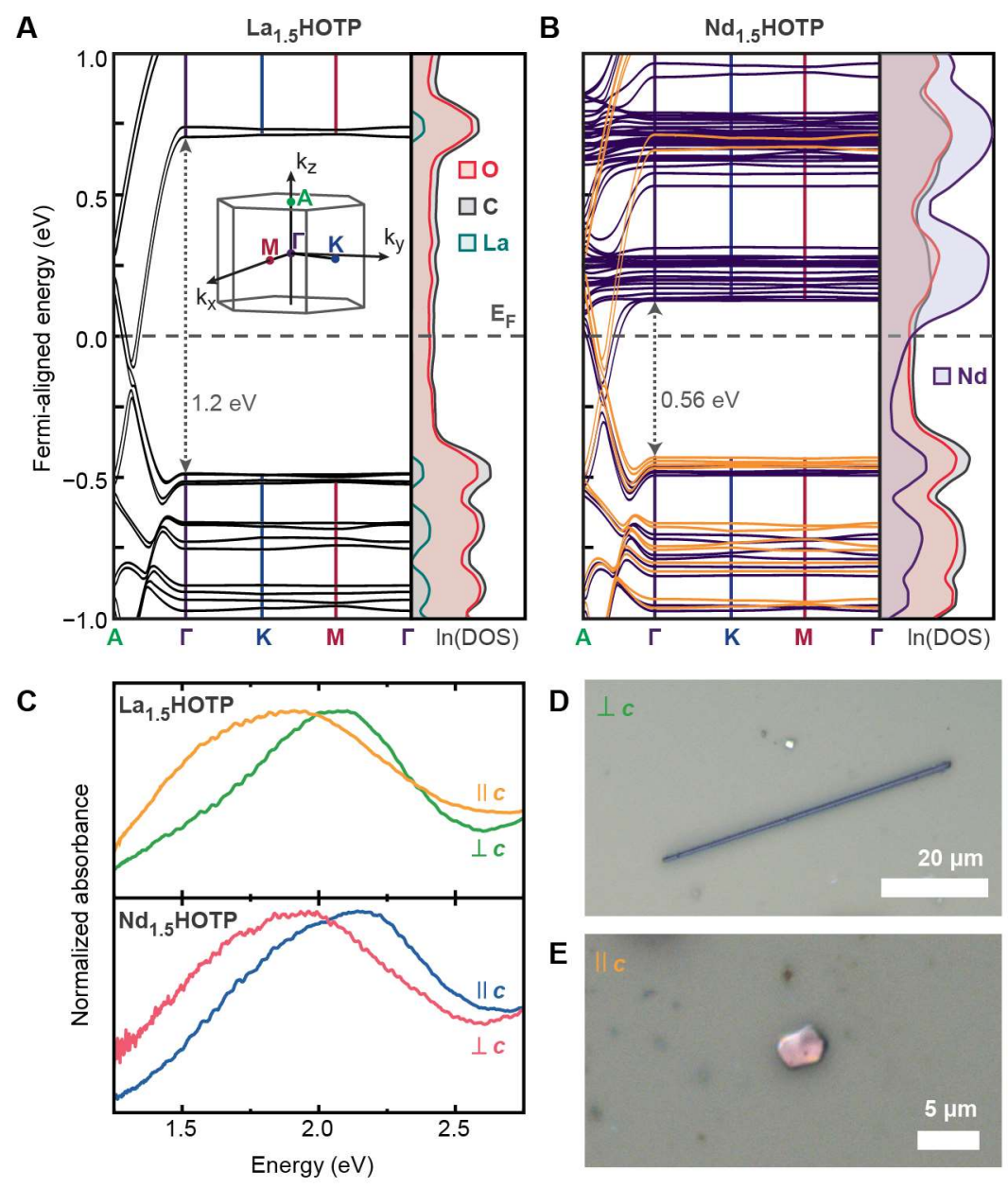

Fig. 2. Electronic structure of $\operatorname{Ln}_{1.5}$ HOTP. (A-B) From density functional theory calculations, both La 1.5 $_{\text {HOTP }}$ (A) and $\mathbf{N d}_{1.5}$ HOTP (B) are metals, with considerable dispersion along the $\mathrm{A}-\Gamma$ direction, originating from the strong $\pi-\pi$ stacking interactions; and essentially flat bands along $\Gamma-\mathrm{K}-\mathrm{M}$. The addition of the partially occupied $4 \mathrm{f}$ orbitals of $\mathrm{Nd}^{3+}$ causes $\mathbf{N d} \mathbf{d}_{1.5}$ HOTP to have a ferromagnetic ground state ((B) shows different spin bands with different colors) and adds a significant number of flat, mostly Ndoriginated bands above the Fermi level. (C) Optical transmission measurements, performed on cleaved microscopic crystals of both materials qualitatively agree with the calculated band structures: when measured along the $c$ direction, the crystals show significantly broader and redshifted absorption features than when measured along the ab planes. (D-E) Examples of the crystals used for the measurements presented on panel (C).

$\Gamma-\mathrm{K}-\mathrm{M}$ directions, where the Fermi level lies within a gap. However, the structure features extensive $\pi-\pi$ stacking in one-dimension along the crystallographic $c$ direction, where the particularly short stacking distance between neighboring organic linkers enables an efficient pathway for charge delocalization and formation of disperse bands.

Optical properties of the materials allow direct confirmation of the electronic anisotropy. Visible to near-infrared absorbance spectra of single crystals of Ln1.5HOTP (Fig. 2A-B) measured orthogonal to the $c$ direction reveal an absorption maximum around 2.1-2.3 eV. Along the $c$ 

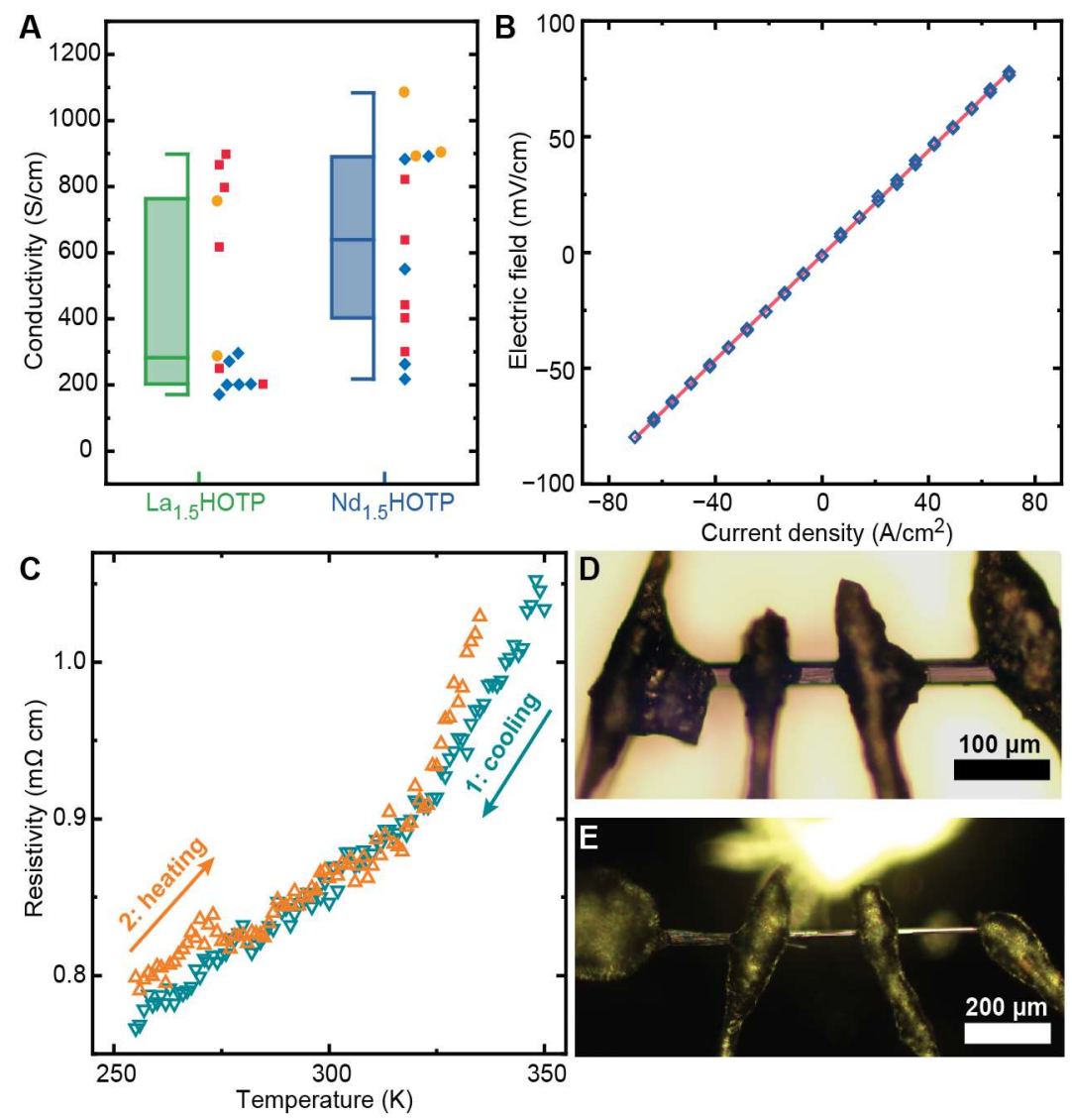

Fig. 3. Electrical transport properties of $\operatorname{Ln}_{1.5} \mathrm{HOTP}$. (A) Both $\operatorname{Ln}_{1.5} \mathrm{HOTP}$ materials showed comparable room-temperature electrical conductivity values, ranging between 170 and $1080 \mathrm{~S} / \mathrm{cm}$, which is considerably higher than the previous record of $150 \mathrm{~S} / \mathrm{cm}$, shown by $\mathrm{Ni}_{3}(2,3,6,7,10,11$ hexaiminotriphenylene $)_{2}(11)$. (B) The conductivity devices showed linear voltage characteristics in a wide range (red line shows linear fit of the data, $\mathrm{R}^{2}=0.999$ ). (C) Between 250 and $350 \mathrm{~K}, \mathbf{N d}_{1.5}$ HOTP showed clear temperature-deactivated conductivity. Devices were fragile and fractured outside this temperature range. (D) Typical variable-temperature device under a bright-field optical microscope. (E) Typical device used for room-temperature measurements under a dark-field optical microscope. Box chart in (A) uses $25 \%-75 \%$ limits for the boxes, minimum and maximum for the whiskers, and median value for the center line. Each symbol in (A) denotes a single-crystal device, with symbols and colors denoting different batches.

direction, the absorption gets considerably broader and the maximum falls below $2 \mathrm{eV}$ in both compounds. Broadening and red-shifting of the absorption features can readily be correlated with an increase in the density of states across the continuum of energy states along the $\mathrm{A}-\Gamma$ vector, compared to the semiconducting gap in the honeycomb plane.

Four-probe single-crystal electrical devices (Fig. 3D-E; Fig. S3) provided clear confirmation of the metallic behavior predicted by DFT. At room temperature, both La1.5HOTP and Nd1.5HOTP showed device-dependent electrical conductivities reaching $900 \mathrm{~S} / \mathrm{cm}$ and $1080 \mathrm{~S} / \mathrm{cm}$, respectively (Fig. 3A). Notably, these values considerably surpass the previous record of electrical conductivity in porous MOFs, $150 \mathrm{~S} / \mathrm{cm}$ shown by $\mathrm{Ni}_{3}(2,3,6,7,10,11 \text {-hexaiminotriphenylene })_{2}(11)$. In fact, to 
our knowledge, this is also the highest electrical conductivity shown by any porous material reported to date. Crystals of Nd 1.5 HOTP show temperature-deactivated conductivity dependence between 250 and $350 \mathrm{~K}$ (fragility of the thin crystals precluded measurements outside this range), a clear characteristic of metallic behavior.

Intriguingly, below $370 \mathrm{~K}$, additional reflections appear in single-crystal diffraction patterns of both La1.5HOTP and Nd1.5HOTP. These new satellite reflections are consistent with the formation of an incommensurate superlattice (Fig. 4A-C). They can be indexed in a monoclinic cell of the same parameters as the structure above $370 \mathrm{~K}$, but require an incommensurate modulation vector of approximately $0.23 c^{*}$ to $0.28 c^{*}$, with slight batch-to-batch and material variation. Raman spectra of single crystals of Ln1.5HOTP (Fig. 4D-E) also exhibit anomalous features at the same transition temperature of approximately 363-368 K. Specifically, we observe clear discontinuities close to the incommensurate transition temperature in the Raman shifts and intensities of several bands associated with the highly anisotropic triphenylene core vibrational modes perpendicular to the crystallographic $c$-axis (17). These may signify a redistribution of charges within the linker and the framework. Importantly, both X-ray and Raman data convey that the structural transition at approximately $370 \mathrm{~K}$ is fully reversible and occurs regardless of the solvation state of either material. Incommensurate modulation is rare in MOFs (18), and so far has only been reported as a guest solvent-dependent phenomenon in electrically insulating systems $(19,20)$, never intrinsic to the framework skeleton. In molecular materials, incommensurate modulation often occurs due to a complex interplay of steric effects and non-covalent interactions (18). In our case, however, evidence suggests that this ordering has an electronic origin.

One-dimensional metals are generally unstable and prone to Peierls distortions. Although a Peierls distortion of an ideal single-atom chain with one electron per atom strictly doubles the unit cell, in the more general case, the Peierls instability leads to the formation of an incommensurately modulated structure known as a CDW (21). The one-dimensional metallic ground state coupled with the temperature-induced formation of an incommensurate superlattice provide strong evidence for assigning the structural transition in Ln 1.5HOTP to CDW ordering. A signature of CDW materials is the characteristic Bardeen-Cooper-Schreiffer (BCS)-like temperature dependence of the energy gap opened by the Peierls transition. This energy gap serves as the order parameter in a CDW system, and is proportional to the square root of the satellite reflection intensities $(21,22)$. Importantly, the intensities of the superlattice reflections ( $\left.\mathrm{I}_{\text {sat }}\right)$ in both Ln1.5HOTP materials are fit well by a BCS-like dependence (Fig. 4C) (here, the fit employs the commonly used gap interpolation formula $\Delta \sim \tanh \left(k \sqrt{1-T / T_{c}}\right)(23-25)$, where $\Delta$ is the gap width, $T$ is the temperature, $T_{\mathrm{c}}$ is the critical temperature where the transition happens, and $k$ is a refined constant). Additional confirmation of our assigning the transition as CDW ordering comes from the behavior of the satellite reflection intensities $\left(I_{\text {sat }}\right)$ near the transition temperature. These follow a polynomial law $\sqrt{I_{\text {sat }}} \sim\left(1-T / T_{c}\right)^{\beta}$, where $\beta$ is the critical exponent (26). The fitting (Fig. S4) provides critical temperatures $T_{c}=361 \mathrm{~K}$ for La1.5HOTP and $T_{c}=365 \mathrm{~K}$ for 

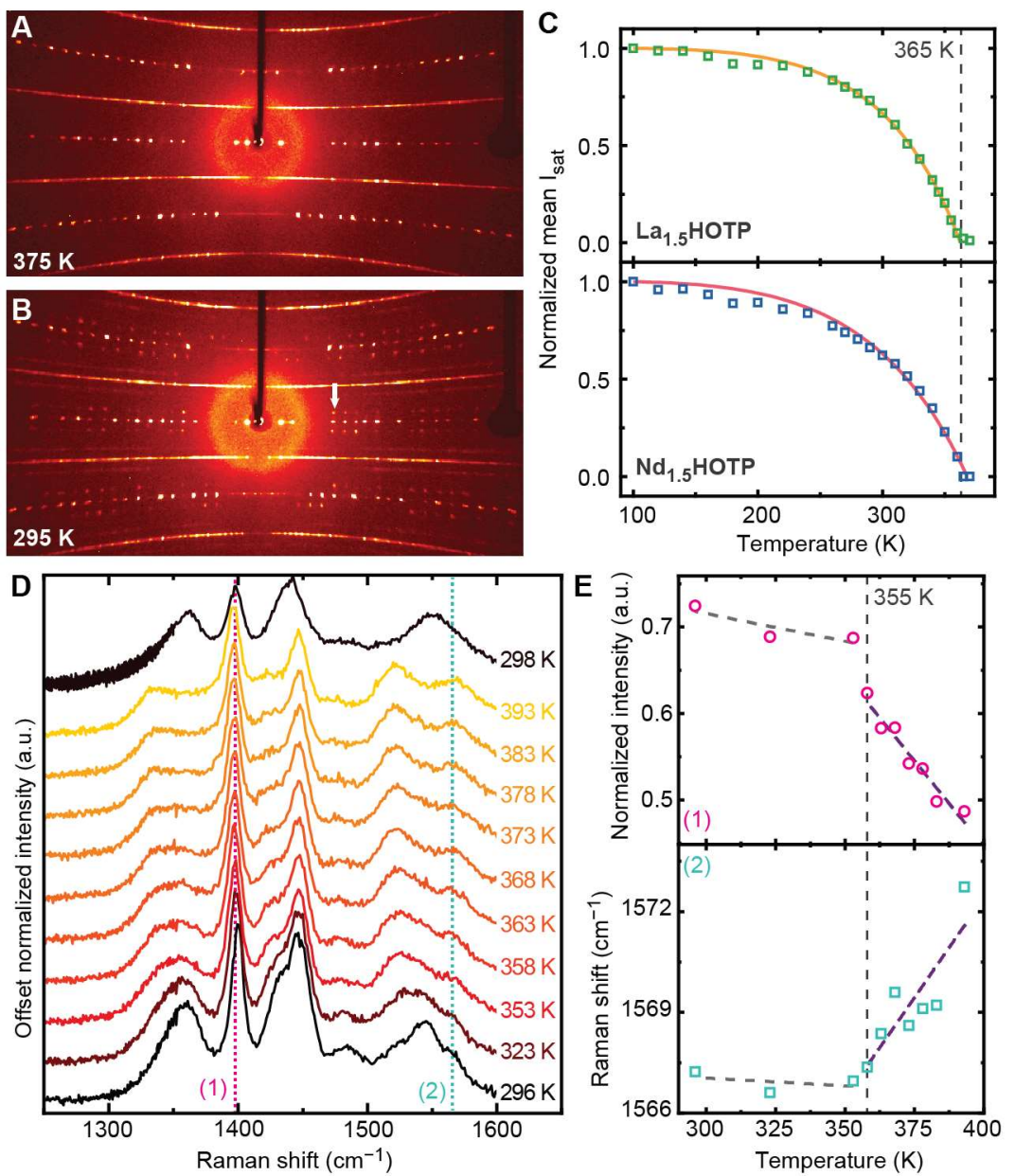

Fig. 4. Charge density wave transition in Ln $\mathbf{n}_{1.5}$ HOTP. (A) High-temperature (above 300-375 K) diffraction data for both materials (here showing Nd $\mathbf{1}_{\mathbf{5}} \mathbf{H O T P}$ ) are fit well by a twinned monoclinic cell, albeit with considerable diffuse scattering. (B) Below the incommensurate transition temperature, both Ln $\mathbf{n}_{1.5} \mathbf{H O T P}$ develop bright satellite reflections that cannot be indexed in any three-dimensional cell. (C) The intensities of the satellite reflections follow a BCS-type dependence: intensities scale with the square of the CDW gap $\Delta$, and its dependence with temperature was calculated using the gap interpolation formula $\Delta \sim \tanh \left(k \sqrt{1-T / T_{c}}\right)$ ), where $\mathrm{k}$ is a refined constant. Solid lines show fit to this dependence and markers show data. (D) Temperature-dependent Raman spectra of Nd $\mathbf{1}_{1.5} \mathbf{H O T P}$ measured with excitation light polarized along the crystallographic $c$ axis possess clear anomalies at the incommensurate transition temperature $(350-360 \mathrm{~K})$. Most prominent are anomalies related to the strong Raman bands in the 1100-1600 $\mathrm{cm}^{-1}$ range, originating from vibrations within the triphenylene core of the linker. (E) Clear discontinuities can be seen in the intensity (top) and Raman shift (bottom) of two bands, indicated in pink and teal on panel (D). Additional Raman data is provided in Figs. S5-S9. Evidently, a change in the phononic structure of the material occurs at $350-360 \mathrm{~K}$.

Nd1.5HOTP, with the same critical exponent of $\beta=1 / 3$, as expected for a second-order transition in the presence of fluctuations (27). This analysis proves that square root satellite intensities behave as a real order parameter in the Ln1.5HOTP systems, which is characteristic of CDW materials (21). Relevantly, a number of other CDW materials exhibit similar critical behavior $(26,28)$, including the well-established example of "blue bronze" $\mathrm{K}_{0.3} \mathrm{MoO}_{3}(29)$. Importantly, a limited set of materials has been conclusively proven to host CDW states, most prominently organic charge- 
transfer salts (30) and low-dimensional metal chalcogenides and oxides (31-34). We emphasize that CDW ordering necessarily originates in metallic systems. This, together with the recordsetting room-temperature conductivity, temperature-deactivated transport, and DFT calculations, provides conclusive proof of the metallic nature of Ln1.5HOTP.

The foregoing results demonstrate metallic conductivity with clear temperature-deactivated transport for Ln 1.5HOTP. The room-temperature conductivity of Nd $\mathbf{1}_{1.5} \mathrm{HOTP}$ exceeds $1000 \mathrm{~S} / \mathrm{cm}$, and is highest among all porous materials. The presence of a charge-density wave transition, exclusively observed in metallic compounds, further substantiates the metallic behavior of the MOFs and points to other potential exotic physical states in these materials. Indeed, we believe, and theoretical studies corroborate (35-39), that other physical phenomena lie undiscovered in these materials - including, perhaps, exotic quantum states and superconductivity. Lowtemperature or high-pressure measurements may lead to the discovery of further anomalies even within the Ln $\mathbf{n}_{\mathbf{1 . 5}} \mathbf{H O T P}$ series.

\section{References and Notes}

1. C. Kittel, Introduction to solid state physics (Wiley, 2004).

2. D. Sheberla, J. C. Bachman, J. S. Elias, C.-J. Sun, Y. Shao-Horn, M. Dincă, Conductive MOF electrodes for stable supercapacitors with high areal capacitance. Nat. Mater. 16, 220-224 (2017).

3. E. M. Miner, L. Wang, M. Dincă, Modular $\mathrm{O}_{2}$ electroreduction activity in triphenylenebased metal-organic frameworks. Chem. Sci. 9, 6286-6291 (2018).

4. C. Liu, F. Li, L.-P. Ma, H.-M. Cheng, Advanced Materials for Energy Storage. Adv. Mater. 22, E28-E62 (2010).

5. E.-K. M. F., S. Veronica, D. Sergey, K. R. B., Laser Scribing of High-Performance and Flexible Graphene-Based Electrochemical Capacitors. Science (80-. ). 335, 1326-1330 (2012).

6. L. S. Xie, G. Skorupskii, M. Dincă, Electrically Conductive Metal-Organic Frameworks. Chem. Rev. 120, 8536-8580 (2020).

7. D. Sheberla, L. Sun, M. A. Blood-Forsythe, S. Er, C. R. Wade, C. K. Brozek, A. AspuruGuzik, M. Dincă, High Electrical Conductivity in $\mathrm{Ni}_{3}(2,3,6,7,10,11-$

hexaiminotriphenylene)2, a Semiconducting Metal-Organic Graphene Analogue. J. Am. Chem. Soc. 136, 8859-8862 (2014).

8. T. Kambe, R. Sakamoto, K. Hoshiko, K. Takada, M. Miyachi, J. H. Ryu, S. Sasaki, J. Kim, K. Nakazato, M. Takata, H. Nishihara, $\pi$-Conjugated nickel bis(dithiolene) complex nanosheet. J. Am. Chem. Soc. 135, 2462-2465 (2013).

9. R. Dong, P. Han, H. Arora, M. Ballabio, M. Karakus, Z. Zhang, C. Shekhar, P. Adler, P. S. Petkov, A. Erbe, S. C. B. Mannsfeld, C. Felser, T. Heine, M. Bonn, X. Feng, E. Cánovas, High-mobility band-like charge transport in a semiconducting two-dimensional metal-organic framework. Nat. Mater. 17, 1027-1032 (2018). 
10. G. Skorupskii, B. A. Trump, T. W. Kasel, C. M. Brown, C. H. Hendon, M. Dincă, Efficient and tunable one-dimensional charge transport in layered lanthanide metalorganic frameworks. Nat. Chem. 12, 131-136 (2020).

11. R. W. Day, D. K. Bediako, M. Rezaee, L. R. Parent, G. Skorupskii, M. Q. Arguilla, C. H. Hendon, I. Stassen, N. C. Gianneschi, P. Kim, M. Dincă, Single Crystals of Electrically Conductive Two-Dimensional Metal-Organic Frameworks: Structural and Electrical Transport Properties. ACS Cent. Sci. 5, 1959-1964 (2019).

12. J. H. Dou, L. Sun, Y. Ge, W. Li, C. H. Hendon, J. Li, S. Gul, J. Yano, E. A. Stach, M. Dincă, Signature of metallic behavior in the metal-organic frameworks $\mathrm{M}_{3}$ (hexaiminobenzene) $2(\mathrm{M}=\mathrm{Ni}, \mathrm{Cu})$. J. Am. Chem. Soc. 139, 13608-13611 (2017).

13. M. E. Foster, K. Sohlberg, M. D. Allendorf, A. A. Talin, Unraveling the Semiconducting/Metallic Discrepancy in $\mathrm{Ni}_{3}(\mathrm{HITP})_{2}$. J. Phys. Chem. Lett. 9, 481-486 (2018).

14. A. J. Clough, J. M. Skelton, C. A. Downes, A. A. De La Rosa, J. W. Yoo, A. Walsh, B. C. Melot, S. C. Marinescu, Metallic Conductivity in a Two-Dimensional Cobalt Dithiolene Metal-Organic Framework. J. Am. Chem. Soc. 139, 10863-10867 (2017).

15. A. J. Clough, N. M. Orchanian, J. M. Skelton, A. J. Neer, S. A. Howard, C. A. Downes, L. F. J. Piper, A. Walsh, B. C. Melot, S. C. Marinescu, Room Temperature Metallic Conductivity in a Metal-Organic Framework Induced by Oxidation. J. Am. Chem. Soc. 141, 16323-16330 (2019).

16. S. A. Cotton, Lanthanide and Actinide Chemistry (John Wiley \& Sons, Ltd, Chichester, UK, 2006).

17. V. Schettino, Infrared and Raman spectra of crystalline triphenylene and triphenylene-d 12 and normal coordinates calculations. J. Mol. Spectrosc. 34, 78-96 (1970).

18. J. J. Oppenheim, G. Skorupskii, M. Dincă, Aperiodic metal-organic frameworks. Chem. Sci. 11 (2020), pp. 11094-11103.

19. G. Chaplais, A. Simon-Masseron, F. Porcher, C. Lecomte, D. Bazer-Bachi, N. Bats, J. Patarin, IM-19: a new flexible microporous gallium based-MOF framework with pressureand temperature-dependent openings. Phys. Chem. Chem. Phys. 11, 5241 (2009).

20. J. Li, Z. Zhou, X. Han, X. Zhang, Y. Yan, W. Li, G. L. Smith, Y. Cheng, L. J. McCormick McPherson, S. J. Teat, M. D. Frogley, S. Rudić, A. J. Ramirez-Cuesta, A. J. Blake, J. Sun, M. Schröder, S. Yang, Guest-controlled incommensurate modulation in a meta-rigid metal-organic framework material. J. Am. Chem. Soc. 142, 19189-19197 (2020).

21. G. Grüner, Density Waves in Solids (CRC Press, Boca Raton, ed. 1, 1994).

22. G. Grüner, The dynamics of charge-density waves. Rev. Mod. Phys. 60, 1129-1181 (1988).

23. K. Senapati, M. G. Blamire, Z. H. Barber, Spin-filter Josephson junctions. Nat. Mater. 10, 849-852 (2011).

24. S. A. Sergeenkov, Electric Field Dependence of the Thermal Conductivity of a Granular Superconductor: Giant Field-Induced Effects Predicted. JETP Lett. 76, 170-174 (2002). 
25. F. Gross, B. S. Chandrasekhar, D. Einzel, K. Andres, P. J. Hirschfeld, H. R. Ott, J. Beuers, Z. Fisk, J. L. Smith, Anomalous temperature dependence of the magnetic field penetration depth in superconducting $\mathrm{UBe}_{13}$. Zeitschrift für Phys. B Condens. Matter. 64, 175-188 (1986).

26. P. Hofmann, M. M. Ugeda, A. Tamtögl, A. Ruckhofer, W. E. Ernst, G. Benedek, A. J. Martínez-Galera, A. Stróżecka, J. M. Gómez-Rodríguez, E. Rienks, M. F. Jensen, J. I. Pascual, J. W. Wells, Strong-coupling charge density wave in a one-dimensional topological metal. Phys. Rev. B. 99, 035438 (2019).

27. T. Ma, S. Wang, Phase transition dynamics (Springer New York, 2014).

28. G. Brusdeylins, C. Heimlich, J. G. Skofronick, J. P. Toennies, R. Vollmer, G. Benedek, L. Miglio, He-atom scattering study of the temperature-dependent charge-density-wave surface structure and lattice dynamics of 2H-TaSe 2 (001). Phys. Rev. B. 41, 5707-5716 (1990).

29. S. Girault, A. H. Moudden, J. P. Pouget, Critical x-ray scattering at the Peierls transition of the blue bronze. Phys. Rev. B. 39, 4430-4434 (1989).

30. D. Jérome, H. J. Schulz, Organic conductors and superconductors. Adv. Phys. 31, 299-490 (1982).

31. G. Travaglini, P. Wachter, J. Marcus, C. Schlenker, The blue bronze $\mathrm{K}_{0.3} \mathrm{MoO}_{3}$ : A new one-dimensional conductor. Solid State Commun. 37, 599-603 (1981).

32. J. A. Wilson, F. J. Di Salvo, S. Mahajan, Charge-density waves and superlattices in the metallic layered transition metal dichalcogenides. Adv. Phys. 24, 117-201 (1975).

33. R. M. Fleming, C. C. Grimes, Sliding-mode conductivity in $\mathrm{NbSe}_{3}$ : Observation of a threshold electric field and conduction noise. Phys. Rev. Lett. 42, 1423-1426 (1979).

34. A. Zettl, C. M. Jackson, A. Janossy, G. Grüner, A. Jacobsen, A. H. Thompson, Charge density wave transition and nonlinear conductivity in NbS3. Solid State Commun. 43, 345-347 (1982).

35. J. L. Mancuso, A. M. Mroz, K. N. Le, C. H. Hendon, Electronic Structure Modeling of Metal-Organic Frameworks. Chem. Rev. 120, 8641-8715 (2020).

36. M. Wu, Z. Wang, J. Liu, W. Li, H. Fu, L. Sun, X. Liu, M. Pan, H. Weng, M. Dincă, L. Fu, J. Li, Conetronics in 2D metal-organic frameworks: Double/half Dirac cones and quantum anomalous Hall effect. 2D Mater. 4, 015015 (2017).

37. J. Li, R. Wu, Metal-organic frameworks: Possible new two-dimensional magnetic and topological materials. Nanoscale. 12, 23620-23625 (2020).

38. L. Z. Zhang, Z. F. Wang, B. Huang, B. Cui, Z. Wang, S. X. Du, H. J. Gao, F. Liu, Intrinsic Two-Dimensional Organic Topological Insulators in Metal-Dicyanoanthracene Lattices. Nano Lett. 16, 2072-2075 (2016).

39. Z. F. Wang, N. Su, F. Liu, Prediction of a Two-Dimensional Organic Topological Insulator. Nano Lett. 13, 2842-2845 (2013). 
Acknowledgments: The authors acknowledge Dr. Lilia S. Xie for helpful discussions and Dr. Vaclav Petricek for advice on X-ray crystal structure refinement of the complex twins. MQA and DLC acknowledge the Laser Spectroscopy Labs at UC Irvine and its director, Dr. Dmitry Fishman, for Raman spectroscopy instrumental support.

Funding: Army Research Office grant W911NF-17-1-0174 (MD)

National Science Foundation grants DMR-1956403 and ACI-1548562 XSEDE (CHH)

\section{Author contributions:}

Conceptualization: GS, MD

Formal analysis: GS, KL, LY, DLC, MQA, MD

Funding acquisition: MQA, $\mathrm{CHH}, \mathrm{MD}$

Investigation: GS, KL, DLC, LY, TC, MQA

Methodology: GS, KL, LY, MQA, CHH, MD

Project administration: MD

Supervision: MQA, CHH, MD

Validation: GS, KL, DLC, LY, MQA, CHH, MD

Visualization: GS

Writing - original draft: GS, MQA, MD

Writing - review \& editing: GS, KL, DLC, LY, TC, MQA, CHH, MD

Competing interests: Authors declare that they have no competing interests.

Data and materials availability: Crystal structure data have been deposited in the Cambridge Structural Database (deposition numbers 2104596-2104597). 


\section{Supplementary Materials:}

\section{Materials and Methods}

Materials

$\mathrm{La}\left(\mathrm{NO}_{3}\right)_{3} \cdot 6 \mathrm{H}_{2} \mathrm{O}$ (Alfa Aesar, 99.9\%), $\mathrm{Nd}\left(\mathrm{NO}_{3}\right)_{3} \cdot 6 \mathrm{H}_{2} \mathrm{O}$ (Alfa Aesar, 99.9\%), N,N'dimethylacetamide (DMA; Sigma Aldrich, 99\% zerO $\left.{ }_{2}\right)$ were used as received. Methanol $(\mathrm{MeOH}$; Fisher, low water, $\geq 99.8 \%$ ) and dichloromethane (DCM; Sigma Aldrich, for HPLC, $\geq 99.9 \%$ ) were dried and deoxygenated using a Glass Contour Solvent Purification System. Reagent-grade water (Millipore Type 1, 18.2 M $\Omega$-cm resistivity) was deoxygenated by bubbling with $\mathrm{N}_{2}$ for at least 24 hours. Hexahydroxytriphenylene $\left(\mathrm{H}_{6} \mathrm{HOTP}\right.$; ACROS Organics, 98+\%) was recrystallized twice from hot DMA (ACS grade, ACROS Organics) and then washed with deionized water (deaerated by bubbling $\mathrm{N}_{2}$ for at least an hour), collected by centrifugation and dried in vacuo. Alternatively, when commercial $\mathrm{H}_{6}$ HOTP was deemed to be of unsatisfactory quality, it was synthesized following a reported procedure (nuclear magnetic resonance spectrum confirming purity of the ligand is shown on Fig. S1) (1). MOF synthesis and reaction work-up, including solvent washes, were performed in an Innovative Technology glovebox with a $\mathrm{N}_{2}$ atmosphere. Most measurements after the washes were performed in air, and the material was kept in aerated solvents. Select manipulations on dry powders as described below were performed under a dry $\mathrm{N}_{2}$ atmosphere in a custom designed MBraun UnilabPro glovebox.

Typical synthesis of Ln1.5HOTP $(\mathrm{Ln} \equiv \underline{\mathrm{La}}, \underline{\mathrm{Nd})}$.

In an $\mathrm{N}_{2}$-filled glove box, $19.5 \mathrm{mg} \mathrm{H}_{6} \mathrm{HOTP}$ (0.060 mmol, 1 equiv) was dissolved in 0.7 $\mathrm{ml} \mathrm{DMA}$ with heating to $90^{\circ} \mathrm{C}$ to produce a yellow solution (blue in case of synthesized $\mathrm{H}_{6} \mathrm{HOTP}_{\text {). }}$ Then, $4156.9 \mathrm{mg}$ of $\mathrm{La}\left(\mathrm{NO}_{3}\right)_{3} \cdot 6 \mathrm{H}_{2} \mathrm{O}$ or $4208.1 \mathrm{mg}$ of $\mathrm{Nd}\left(\mathrm{NO}_{3}\right)_{3} \cdot 6 \mathrm{H}_{2} \mathrm{O}$ (9.600 mmol, 160 equiv) was dissolved in $6 \mathrm{ml}$ of deoxygenated water by heating to $90^{\circ} \mathrm{C}$. The linker solution was then combined with the solution of the metal salt to produce a pale yellow (La) or pink (Nd) solution, which was then passed through a $200 \mathrm{~nm}$ polyethersulfone filter and transferred into a $35 \mathrm{ml}$ capacity glass pressure tube (Synthware). For improved crystallinity and yield, borosilicate glass rods were sanded with 220 grit sandpaper, cleaned by sonication in water and methanol, and added to the reaction mixture in order to provide nucleation sites for the crystals. After that, the pressure tube was fitted with a PTFE screw plug with a back-seal silicone O-ring (Synthware), taped with electrical tape $(3 \mathrm{M})$, and placed inside a preheated oven set to $135^{\circ} \mathrm{C}$. The mixture is then kept at that temperature for 72 hours, after which the oven is allowed to cool down to room temperature over 8 hours. The tube is then transferred into a $\mathrm{N}_{2}$-filled glove box, where it is opened, and the reaction is worked up by removing the solution using a pipette and transferring the crystals into clean solvent. The crystals are washed by soaking in deoxygenated water four times with at least one overnight soak, and no soaks shorter than one hour. The crystals are then similarly soaked in deoxygenated methanol, and, in some cases, with dichloromethane. The crystals can then be stored in air under methanol, dichloromethane, or diethyl ether without observed changes in crystal quality or electrical conductivity over the course of at least several months. 
Elemental analysis and formula determination.

Elemental analysis was performed by Robertson Microlit Laboratories, Ledgewood NJ. Samples were activated at $90^{\circ} \mathrm{C}$ for 24 hours in vacuo on a Schlenk line and handled in dry $\mathrm{N}_{2}-$ filled gloveboxes. Carbon, hydrogen and nitrogen content were obtained using conventional CHN analysis. Metal content was obtained using inductively coupled plasma optical emission spectroscopy.

Due to significant disorder, it was impossible to determine crystallographically whether the metal nodes were solvated with aquo or hydroxo ligands. Thus, the formula for the crystal was set following the results of density functional theory (DFT) calculations, where reasonable electronic structures were obtained only when all HOTP linkers were in their -3 charge states, that is, with one hydroxyl adding an additional negative charge per metal ion, making the overall framework formula $\left[\mathrm{Ln}(\mathrm{OH})\left(\mathrm{OH}_{2}\right)\right]_{1.5} \mathrm{HOTP}$.

La1.5HOTP: $\left[\mathrm{La}(\mathrm{OH})\left(\mathrm{OH}_{2}\right)\right]_{1.5} \mathrm{HOTP} \cdot 2.6 \mathrm{H}_{2} \mathrm{O} \cdot 1.5 \mathrm{MeOH} \cdot 0.3 \mathrm{DMA}$

(calcd., found for $\left.\mathrm{La}_{1.5} \mathrm{C}_{20.7} \mathrm{H}_{24.3} \mathrm{~N}_{0.3} \mathrm{O}_{13.4}\right)$ : $\mathrm{C}(35.52,35.52), \mathrm{H}(3.50,3.53), \mathrm{N}(0.58,0.58)$, $\operatorname{La}(29.81,29,82)$

Nd1.5HOTP: $\left[\mathrm{Nd}(\mathrm{OH})\left(\mathrm{OH}_{2}\right)\right]_{1.5} \mathrm{HOTP} \cdot 0.2 \mathrm{H}_{2} \mathrm{O} \cdot 0.8 \mathrm{DCM} \cdot 0.1 \mathrm{Nd}\left(\mathrm{NO}_{3}\right)_{3}$

(calcd., found for $\left.\mathrm{Nd}_{1.6} \mathrm{C}_{18.8} \mathrm{H}_{12.5} \mathrm{~N}_{0.3} \mathrm{O}_{10.0} \mathrm{Cl}_{1.7}\right)$ : $\mathrm{C}(32.63,32.63), \mathrm{H}(1.82,1.80), \mathrm{N}(0.60$, $0.56), \mathrm{Nd}(32.26,32.27)$

Powder $\underline{X-r a y}$ diffraction.

Laboratory powder X-ray diffraction (PXRD) patterns were collected on a Bruker D8 diffractometer fitted with a Goebel mirror and a Lynxeye Si strip position-sensitive detector, operating in reflection mode with Ni-filtered $\mathrm{Cu} \mathrm{K} \alpha_{1,2}$ radiation $\left(\mathrm{K} \alpha_{1}=1.5406 \AA\right.$, $\mathrm{K} \alpha_{2}=1.5444$ $\left.\AA, K \alpha_{2} / K \alpha_{1}=0.5\right)$. Samples were prepared by placing a thin layer of the appropriate material on a zero-background silicon crystal plate.

PXRD patterns (Fig S2) of the materials indicate excellent crystallinity and confirm overall phase purity of the samples. Weak impurity peaks are sometimes observed, primarily at $4.6^{\circ} 2 \theta$, belonging to a previously reported cubic phase (2).

$\underline{\text { Single crystal }} \underline{X \text {-ray diffraction. }}$

Single-crystal diffraction data were collected on a Bruker-AXS D8 Venture Kappa Duo diffractometer coupled to a Photon III CPAD detector, using Mo K $\alpha$ radiation $(\lambda=0.71073 \AA)$ from an $\mathrm{I} \mu \mathrm{S} 3.0$ microfocus source, performing $\varphi$-and $\omega$-scans. Generally, dynamic sensing mode was used. For preliminary measurements, crystals of the materials were taken from mother liquor, methanol, DCM suspensions, or taken first allowed to dry on a glass slide, and then transferred into Paratone oil and mounted on crystal loops (MiTeGen). No qualitative differences were found in the low-temperature $(100 \mathrm{~K})$ diffraction, with crystals from all solvents, as well as desolvated crystals, providing incommensurately modulated cells with similar q-vectors. Full lowtemperature datasets were obtained in the same manner for both $\mathrm{Ln}_{1.5} \mathrm{HOTP}$ materials. Although 
stable at room temperature and below, we have found that unprotected crystals sometimes degrade fast in the $\mathrm{N}_{2}$ cryostream at temperatures above $\sim 350 \mathrm{~K}$, depending on the crystals size and thickness of the oil coating. Thus, for variable-temperature and high-temperature measurements we sealed the crystals in capillaries: the crystal was first screened at $100 \mathrm{~K}$ to ensure good diffraction. Then, the crystal covered in a small amount oil was transferred inside a thin polyimide capillary glued to a thin stainless-steel rod with fast-curing two-component epoxy. The rod with the capillary was then secured on a goniometer mount and evacuated on a Schlenk line for 30 minutes in order to remove air bubbles from the oil. The sample was then transferred into a dry $\mathrm{N}_{2}$-filled glove box, where the top of the capillary was sealed with fast-curing two component epoxy. No significant degradation was observed in thus sealed crystals held for several days above $370 \mathrm{~K}$. To obtain the graphs in Fig. 4, crystals in sealed capillaries were first cooled down to 100 $\mathrm{K}$, then 45 - or 90 -degree $\varphi$ scans were performed at each presented temperature. These patterns were then integrated with SAINT (Bruker-AXS) and scaled with SADABS (Bruker-AXS). 90-120 strong main reflections and 90-120 strong satellite reflections were then picked so they are observed at all temperatures, and mean satellite reflection intensity for those reflections is then normalized by mean main reflection intensity to obtain the curves in Fig. 4. Individual satellite reflections when normalized by mean main reflection intensity generally follow the same temperature dependence as presented in Fig. 4, but with more noise, as would be expected.

High-temperature diffraction data were integrated using SAINT, and absorption correction and scaling were performed using SADABS. The SHELX suite (3) was used for structure solution and refinement, and JANA2020 (4) was used for final refinement of the heavily twinned data. For $\mathrm{Nd}_{1.5}$ HOTP, the structure was integrated and in the monoclinic C-centered cell described in the CIF file and below. The structure was solved using SHELXT (5) and refined against $\mathrm{F}^{2}$ on all data by full-matrix least squares with SHELXL. Significant violations of the C-centering absences were found in the data, however, attempts to refine the structure in a lower-symmetry cell proved unsuccessful, and good residuals were obtained for space group $\mathrm{C} 2 / \mathrm{c}$ with strong six-component pseudo-merohedral twinning. For La1.5HOTP, the data were first integrated in a monoclinic primitive cell, and solved using SHELXT, and a rough model was obtained by refinement in SHELXL. The data were then re-integrated in a hexagonal supercell which fit all twinned monoclinic domains, with dimensions of $a=44.0 \AA, c=6.1 \AA$. This dataset was then input into JANA2020 along with the initial model from SHELXL, where the final refinement was performed against $\mathrm{F}^{2}$ on all data from the six twinned domains in space group $\mathrm{P} 2{ }_{1} / \mathrm{n}$. All non-hydrogen framework atoms were refined anisotropically, and coordinated oxygens were refined anisotropically except for several atoms where good convergence was not achieved for anisotropic models. Pore solvent oxygen atoms (presumably, belonging to water molecules) were all refined isotropically. Hydrogen atoms were refined using the riding model, and only for carbon atoms as no clear maxima were observed in the residual density for water protons. Selected information regarding the data collection and refinement are presented in Table S1.

Electrical conductivity measurements 
Electrical conductivity was measured using the linear four-contact probe method (6). Devices (Fig. S3) were fabricated in air by carefully contacting the crystals with thin wires wetted with water-based highly conductive graphite adhesive (EMS). Silver-based adhesives were tested but provided significantly higher contact resistances. Humidity was controlled during the device fabrication process and kept between $40-55 \%$ relative humidity to reduce static charging. Generally, $12.5 \mu \mathrm{m}$ copper wires were used for devices intended for room-temperature measurements. Contact resistances, as determined from two-wire measurements of the same devices, were generally large. Current contacts generally were in the $10^{4} \Omega$ range, and voltage contacts frequently had even higher resistances, reaching into the $10^{5} \Omega$ range. This difference between current and voltage contacts did not appear to depend on the size of the contacts, so we believe the likely explanation is that current contacts would usually contact the top and bottom ends of the crystal (the $a b$ plane), allowing polarization of the full thickness of the crystal; voltage contacts, however, can only contact the sides ( $a c, b c$, and equivalent), and due to the strong structural anisotropy, only a small layer of the crystal would be polarized. Only larger $(>50 \mu \mathrm{m}$ in thickness) crystals of $\mathrm{Nd}_{1.5}$ HOTP produced well-functioning variable-temperature devices, and smaller crystals would either break easily, or have poor contact. No crystals of La1.5HOTP large enough for variable temperature measurements were obtained. Devices intended for variabletemperature measurements were made with thicker $(25 \mu \mathrm{m})$ gold wires to ensure good contact. Contact resistances for these crystals were in the $10^{2} \Omega$ range for current contacts and $10^{3}-10^{4}$ range for voltage contacts. Dimensions of the devices were measured using a Nikon SMAZ-745T optical microscope.

Room-temperature measurements were carried out using a Janis ST-500 probe station. Crystals contacted with thin wires were put on glass slides; wires contacting the crystals were then connected with graphite adhesive to copper tape pads, which were used to contact the devices with gold-plated tungsten probe tips. In some cases, to reduce crowding, $100 \mu \mathrm{m}$ copper wires were used in-between the thinner wires contacting the crystal and the copper tape pads. I-V curves were then measured using a Keithley 2450 sourcemeter connected to the probes with triaxial cables. Current limits were generally between $\pm 0.1 \mathrm{~mA}$, although thicker $(>60 \mu \mathrm{m})$ crystals were often stable up to 1-2 mA. Two-probe resistances were checked most often with $\pm 0.1 \mathrm{~V}$ limits. A significant number of devices showed poor contact with nonlinearities around zero applied current, and thus were not included in the conductivity statistics. Only devices which produced clear linear (Ohmic) I-V behaviour were included in the conductivity statistics. Measurements were performed in air; temperature was generally at $21^{\circ} \mathrm{C}$, and the humidity was between $10-20 \%$ relative humidity.

Variable temperature measurements were conducted using a Quantum Design Physical Property Measurement System (PPMS) Dynacool 9 T, using the Electrical Transport Option as configured by the manufacturer. For variable-temperature measurements, devices were put on pieces of sapphire wafers; then the thin gold wires contacting the crystals were attached with waterbased graphite adhesive to thicker $(100 \mu \mathrm{m})$ copper wires, which were soldered to the PPMS 
resistivity puck. Apiezon N-grease was used to enhance thermal contact between the puck and the sapphire chip. Measurements were performed in a low pressure of He (5-10 torr). After initial evacuation devices did not see significant changes in resistance when held at a constant temperature under a low pressure of $\mathrm{He}$ or in high-vacuum conditions. Variable-temperature resistivities of the crystals were determined using the low-frequency lock-in method, with excitation frequencies between $0.3 \mathrm{~Hz}$ and $3 \mathrm{~Hz}$; and excitation amplitudes generally between 0.05 $\mathrm{mA}$ and $0.2 \mathrm{~mA}$. I-V curves were collected to confirm Ohmic behaviour up to at least $20 \%$ higher current than the highest used excitation amplitude for resistivity measurements. Phase angle was constantly monitored during the measurement, and excitation frequency was adjusted to ensure the phase angle remained within $\pm 5^{\circ}$. Prior to the measurement, devices were screened to ensure they showed good Ohmic contact and room temperature resistivities within the range shown on Fig. 4A. We fabricated three independent devices of Nd1.5HOTP that satisfied the above requirements, and all showed thermally deactivated conductivity. Devices were first cooled down to 260-270 K, then heated to $330-350 \mathrm{~K}$, then cool-heat cycles were repeated several times with expanded temperature limits until the crystal broke. Thermally deactivated transport was observed consistently until the crystal got damaged, which was evident in irreversibly increased resistance and/or high phase angles. Such damaged crystals formed large cracks or split into several parts.

Density functional theory calculations

Crystallographic high-temperature structures were used as starting points for calculations. Minority disorder components were removed, resulting in hexagonal structures. Hydrogen atoms were added using CCDC Mercury (7) to coordinated water and hydroxyl molecules, so that there would be one hydroxo and one aquo ligand per metal atom, in order to ensure charge neutrality for calculations. Several models were assessed, with different charge states of the HOTP linkers and different magnetic interactions for the open-shell Nd1.5HOTP. Specifically, models with overall formulas $\mathrm{Ln}_{6}(\mathrm{HOTP})_{4}\left(\mathrm{H}_{2} \mathrm{O}\right)_{12}$ (giving an average HOTP charge state of -4.5), $\mathrm{Ln}_{6}(\mathrm{HOTP})_{4}\left(\mathrm{H}_{2} \mathrm{O}\right)_{10}(\mathrm{OH})_{2}$ (giving an average HOTP charge state of -4), and $\mathrm{Ln}_{6}(\mathrm{HOTP})_{4}\left(\mathrm{H}_{2} \mathrm{O}\right)_{6}(\mathrm{OH})_{6}$ (giving an average HOTP charge state of -3 ) were evaluated, with antiferromagnetic (AFM), ferromagnetic (FM), and spin-paired calculations performed for $\mathrm{Nd}_{1.5}$ HOTP. Of all these models, only the one with the overall formula $\mathrm{Ln}_{6}(\mathrm{HOTP})_{4}\left(\mathrm{H}_{2} \mathrm{O}\right)_{6}(\mathrm{OH})_{6}$ and FM order gave chemically reasonable band diagrams, whereas the other models resulted in a large number of Nd-originated bands at and around the Fermi level, which would lead to unrealistic f-orbital populations. Thus, the charge state of HOTP was presumed to be -3 , as was also previously reported for related materials (8). All calculations were carried out using DFT as implemented in the Vienna ab initio Simulation Package (VASP, version 5.4.4) (9). All structures were equilibrated using the unrestricted GGA-PBEsol exchange-correlation functional (10). The plane-wave cut off was set to $500 \mathrm{eV}$. Ionic relaxation convergence criteria and electronic convergence criteria was set to $0.005 \mathrm{eV}^{-1}$ and $10^{-6} \mathrm{eV}$, respectively. A $\Gamma$-only k-grid $(1 \times 1 \times 1)$ was used for all optimization due to the large size of the unit cells. Both structures were optimized with unrestricted spin-polarized calculations resulting in a non-magnetic structure for La1.5HOTP and a FM structure for Nd1.5HOTP. The optimizations also resulted in a reduction in the $c$ 
parameter from $6.14 \AA$ to $5.76 \AA$ and $6.05 \AA$ to $5.75 \AA$ for La1.5HOTP and Nd1.5HOTP, respectively.

After the optimized structure were obtained, single-point calculations were performed on these structures with GGA-PBEsol functional (10) to obtain the initial wavefunction for the electronic band structure (EBS) and density of state (DOS) calculations. These single point calculations were performed with a higher resolution k-grid of $2 \times 2 \times 4$. The EBS and DOS for La 1.5 HOTP were obtained with GGA-PBEsol functional (10) and similar convergence criterion as mentioned in the optimization process. For Nd1.5HOTP, a Hubbard U correction was added to properly account for the interactions of $4 f$ valence electrons, using the GGA+U functional $(\mathrm{U}=$ $4.8 \mathrm{eV}, \mathrm{J}=0.6 \mathrm{eV}$; these parameters were obtained from the constrained random-phase approximation studies for early lanthanides) (11). As the optimized cells possessed unit cell $c$ parameters significantly smaller than those obtained experimentally, we investigated the influence of the stacking distance on the band structures (Fig. S10). From the optimized structures of La1.5HOTP and Nd1.5HOTP, we increased the $c$ lattice parameter from the optimized values to the experimentally obtained values. These structures were then optimized with restricted unit cell, and EBS along with DOS for each were computed using similar methods as above to elucidate the effect of interlayer stacking distance on the materials' electronic properties. We found that the band structures were qualitatively similar within each material irrespective of the stacking distance.

Raman spectra for the optimized structure of La1.5HOTP were obtained using Raman offresonant activity calculator coupling with VASP (version 5.4.4) (9). The macroscopic dielectric tensors were obtained with DFT as implemented in VASP via phonon calculations at the zone center ( $\Gamma$-point) with the finite displacements method (FDM). Central difference was enforced to allow all displacements and the step size was set to be $0.01 \AA$. All calculations were performed with GGA-PBEsol functional (10). Visualization of each vibrational mode (Table S3) was obtained by plotting the vibrational vectors associated with the corresponded mode obtain from the $\Gamma$-point phonon calculations.

Optical spectroscopy.

Visible range optical measurements of La1.5HOTP and Nd ${ }_{1.5}$ HOTP single crystals were carried out using a Cytoviva ${ }^{\circledR}$ hyperspectral imaging microscope system equipped with an ImSpector V10E spectrograph, spectral camera, and L1090 MR16 150W Halogen visible light source. Bright-field transmission spectroscopy measurements were performed on in-plane (incident light parallel to the $c$ axis) and out-of-plane (incident light orthogonal to the $c$ axis) oriented La1.5HOTP and Nd1.5HOTP crystals mounted on double sided polished $500 \mu \mathrm{m}$ thick Zcut quartz substrates (University Wafers). Background correction of the transmission hyperspectral micrographs was performed by subtracting the light source and quartz substrate contributions. Background correction, conversion to absorbance units, region of interest averaging, and spectra extraction were performed on raw hyperspectral micrographs using the L3HARRISTM ENVI® data processing software. 


\section{Raman spectroscopy.}

Temperature-dependent Raman spectra were collected on in-plane (incident laser parallel to the $c$ axis) and out-of-plane (incident laser orthogonal to the $c$ axis) oriented La1.5HOTP and Nd1.5HOTP single crystals using a Renishaw Invia Raman microscope equipped with a chargecoupled detector (CCD) capable of measurements up to $1 \mathrm{~cm}^{-1}$ resolution. In these measurements, the crystals were mounted onto $\mathrm{Si} / \mathrm{SiO}_{2}(300 \mathrm{~nm})$ substrates with fiduciary markers. A $532 \mathrm{~nm}$ laser was employed as the illumination source with a nominal power of $0.22 \mathrm{~mW}$ and $10 \mathrm{~s}$ of exposure time. The sample temperature was controlled using a Linkam THMS600 stage purged with pure Ar gas. Raw intensities were normalized, and pertinent sections of the Raman spectra were fit with a cubic spline background and Lorentzian peaks with MagicPlotPro 2.9 software. To compare temperature-dependent behavior of specific vibrational modes, the peak amplitude was used as a measure of intensity and the peak center as a measure of position. The out-of-plane (caxis) crystal orientation was identified from large flat crystal facets while the in-plane crystal ( $a$ axis) crystal orientation was identified from domains of hexagonal platelets.

$\underline{\text { Raman Vibrational Mode Assignments and Phonon Mode Anisotropy }}$

The quasi-one-dimensional crystal structure of La1.5HOTP leads to well resolved and polarized vibrational modes. By combining crystallographic orientation dependent Raman spectroscopy and DFT vibrational mode simulations, we were able to assign distinct and highly anisotropic vibrational modes from both the inorganic unit and the HOTP core. Close inspection of the Raman spectra with the laser polarization parallel to the crystallographic c-axis (incident beam perpendicular to the c-axis) of the micrometer La1.sHOTP crystal reveals the prominence of core phonon modes corresponding to vibrational vectors that lie along the HOTP ligand plane assignable to an A1' symmetry (20). Peaks B, C, F, and H in Fig. S8 and Table S3 are the most dominant peaks in this orientation. Orienting the crystal with the (a) laser polarization perpendicular to the crystallographic $c$-axis (incident beam perpendicular to the $c$-axis) and (b) laser polarization parallel to the $a b$-plane (incident beam parallel to the $c$-axis) resulted to very similar Raman spectra which both exhibited intense peaks that correspond to HOTP vibrational modes with E' symmetry. These peaks represent the A, D, E, and G modes in Fig. S8 and Table S3. Phonon modes that are predominantly oriented along the crystallographic c-axis, such as in peaks $\mathrm{J}$ and $\mathrm{L}$, also become more intense in orientations (a) and (b). The analyses of the temperature-dependent evolution of the Raman phonon modes were derived from these aforementioned peaks, comparing and contrasting only those that have the same vibrational symmetries. 


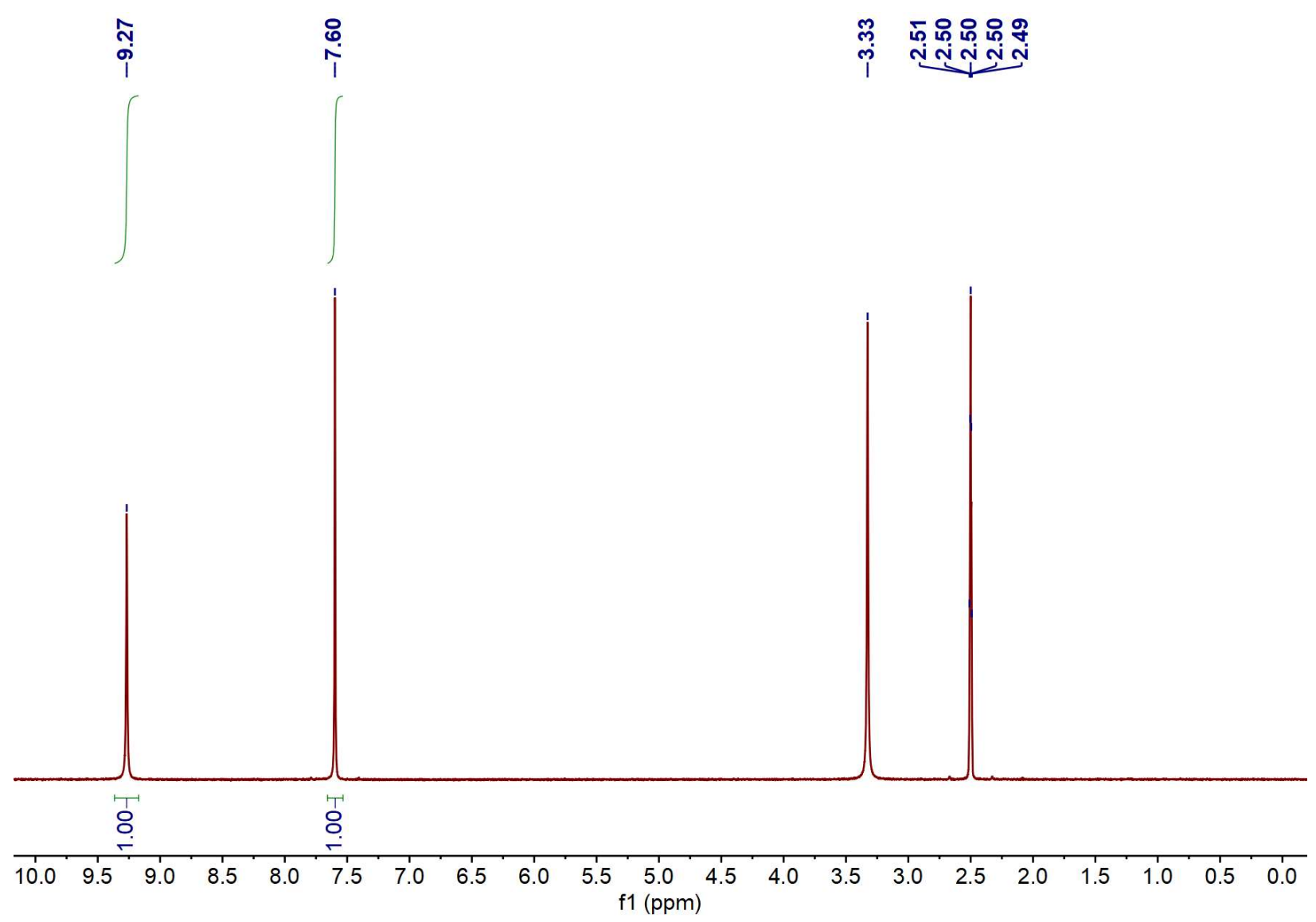

Fig. S1.

Nuclear magnetic resonance spectrum of synthesized $\mathrm{H}_{6} \mathrm{HOTP}$, measured in dimethylsulfoxide$\mathrm{d}_{6}$ on a Bruker Avance-III HD Nanobay spectrometer operating at $400.09 \mathrm{MHz}$. 


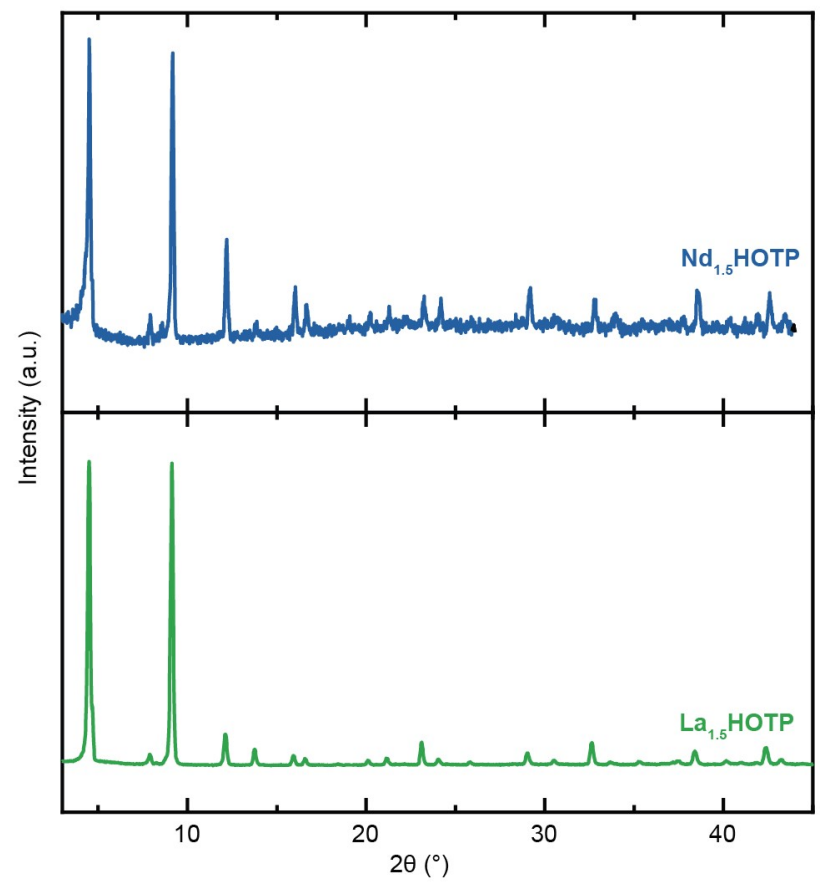

Fig. S2.

Example PXRD patterns of Ln1.5HOTP. 


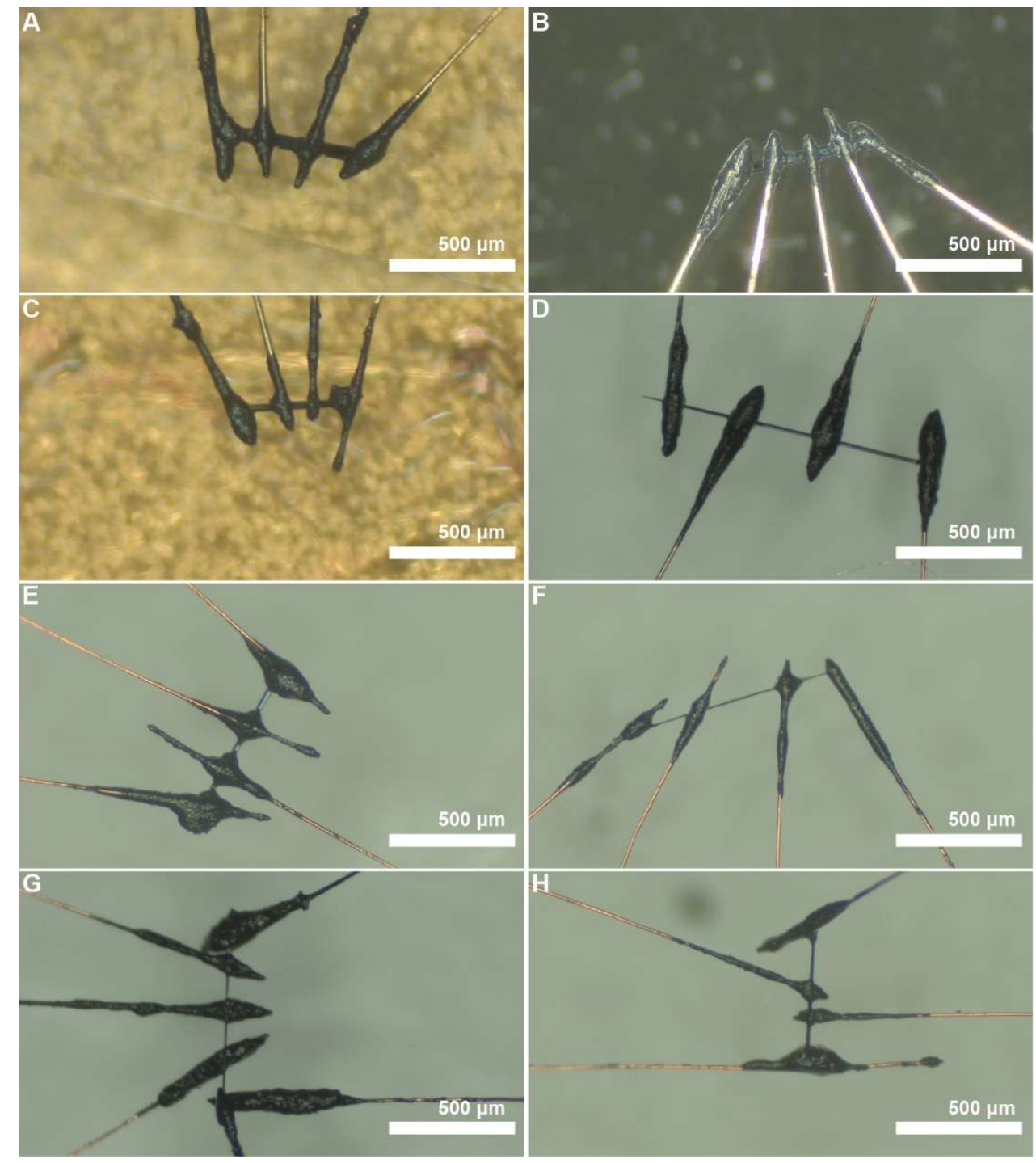

Fig. S3.

Single-crystal conductivity devices of Nd1.5HOTP (A-D) and La1.5HOTP (E-H) used for variable-temperature $(\mathrm{A}, \mathrm{C})$ and room temperature measurements. 


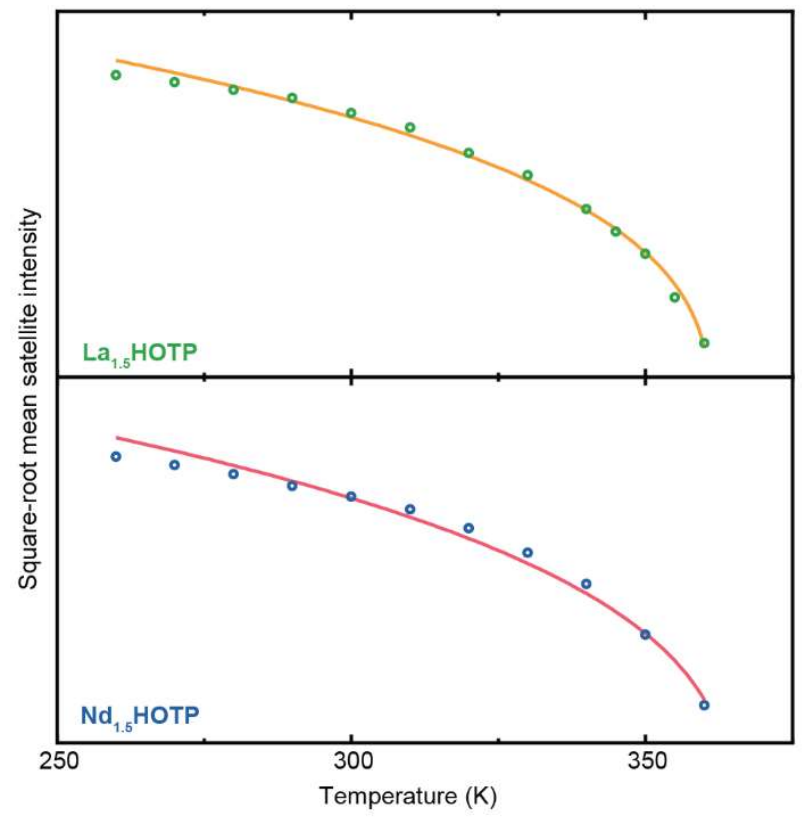

Fig. S4.

Critical exponent analysis of satellite intensities in $\mathrm{Ln}_{1.5}$ HOTP. The square root of the mean satellite intensity for each material (points) fits well to the dependence $\sqrt{I_{\text {sat }}} \sim\left(1-T / T_{c}\right)^{\beta}$, where $\beta=1 / 3$. This is typical behavior for the order parameter near a second-order phase transition in the presence of fluctuations, and has been observed previously for other CDW systems. 

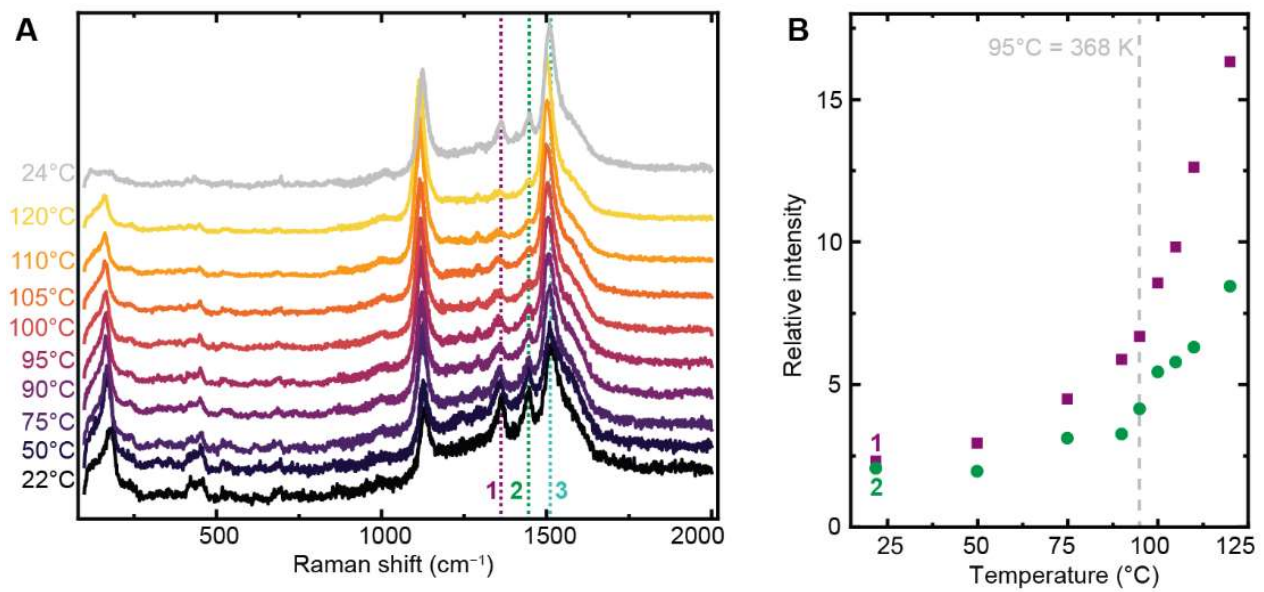

Fig. S5.

Temperature-dependent Raman spectra for La1.5HOTP measured with excitation light polarized parallel to the crystallographic $c$ axis (A) and anomalous behavior of intensities of two prominent peaks with temperature (B) plotted as the ratios of the peak intensity of the strongest band, marked 3, to the other two marked bands. 

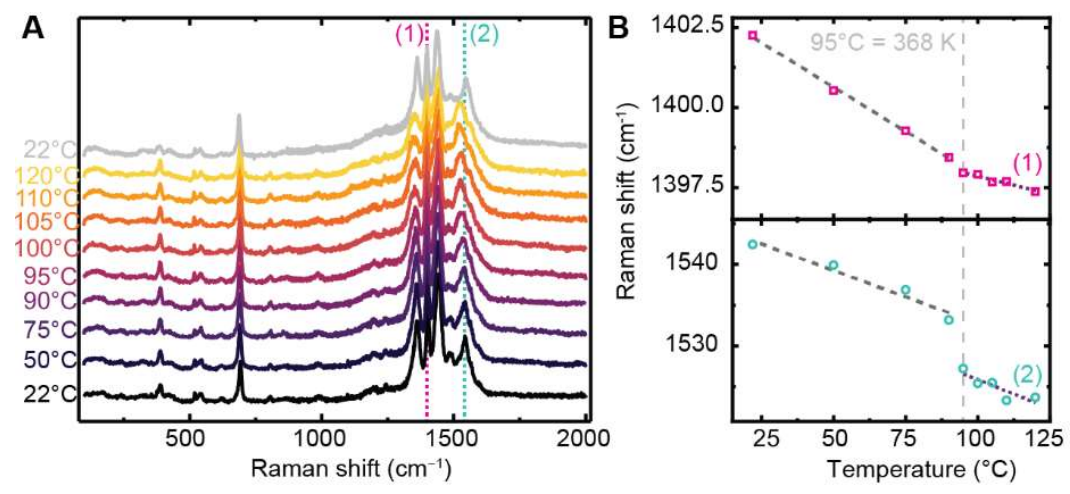

Fig. S6.

Temperature-dependent Raman spectra for La1.5HOTP measured with excitation light polarized orthogonal to the crystallographic $c$ axis (A) and anomalous behavior of the Raman shifts of two prominent peaks with temperature $(\mathrm{B})$. 


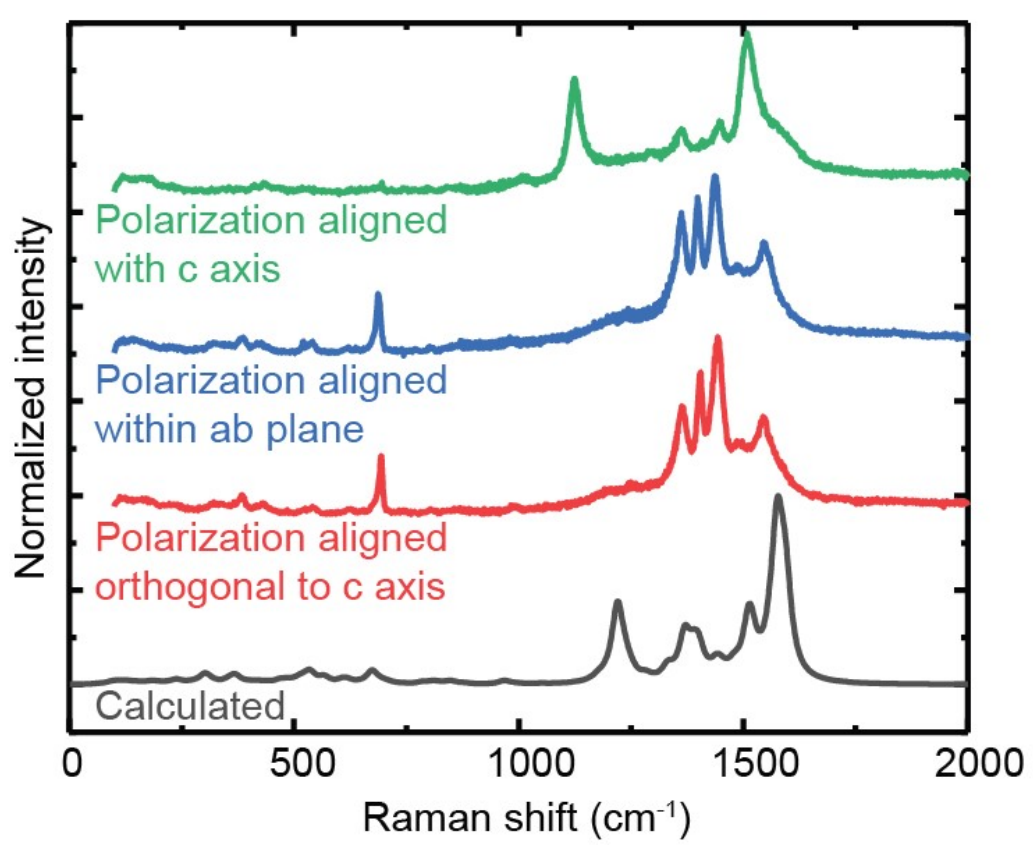

Fig. S7.

Raman spectra for La1.5HOTP: (top, green) with excitation light polarization aligned with the crystal's $c$ axis; (second from top, blue) with excitation light polarization aligned within the ab plane of the crystal; (third from top, red) with excitation light polarization aligned orthogonal to the crystal's $c$ axis; (bottom, grey) calculated using DFT. 


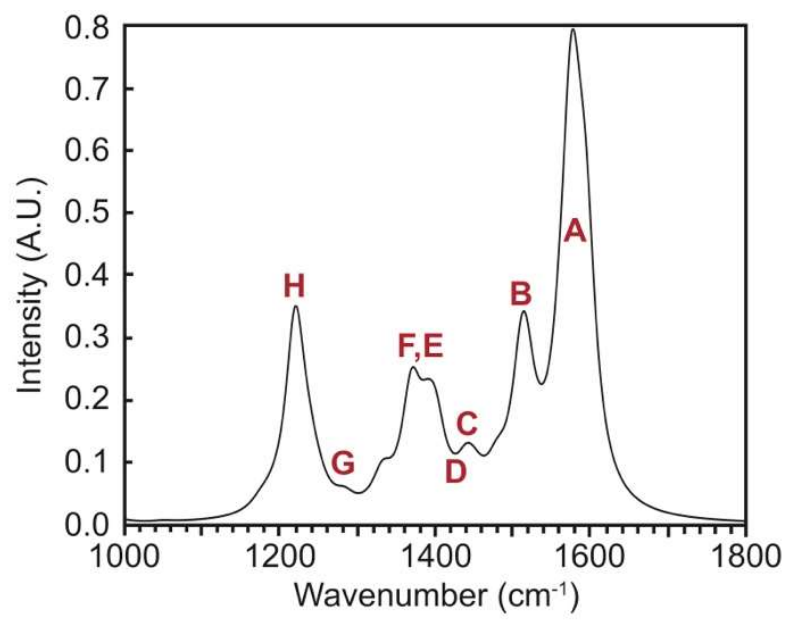

Fig. S8.

Part of simulated Raman spectrum for La1.5HOTP featuring primarily linker-based vibrations. Labels match those shown in Table S3. 


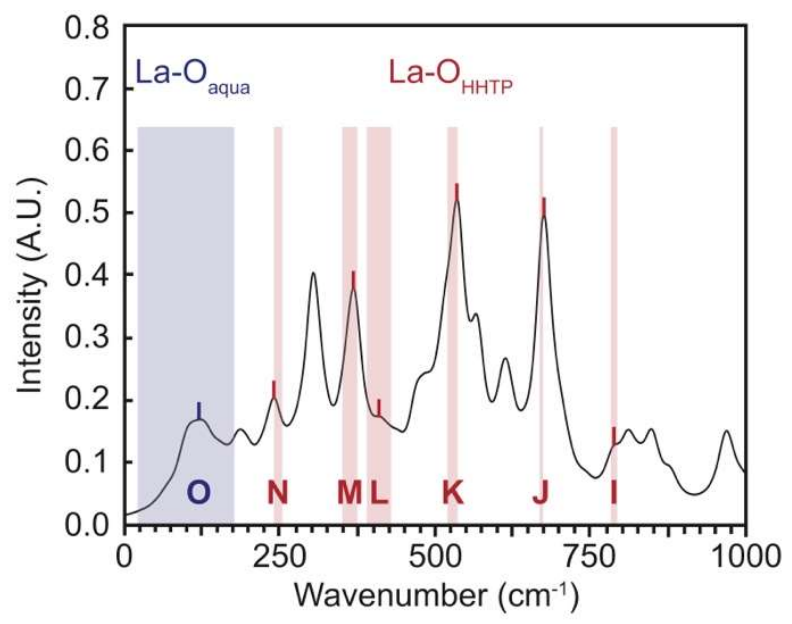

Fig. S9.

Part of simulated Raman spectrum for La1.sHOTP with highlighted La-O vibrations. Labels match those shown in Table S3. Red marks vibrations originating primarily from La and the linker oxygen atoms. Blue marks vibrations originating primarily from La and the aqua ligand oxygen atoms. Frequency for all La-O vibrations: 794-783, 674-669, 536-520, 429-390, 374$350,253-240,177-20 \mathrm{~cm}^{-1}$. 
A
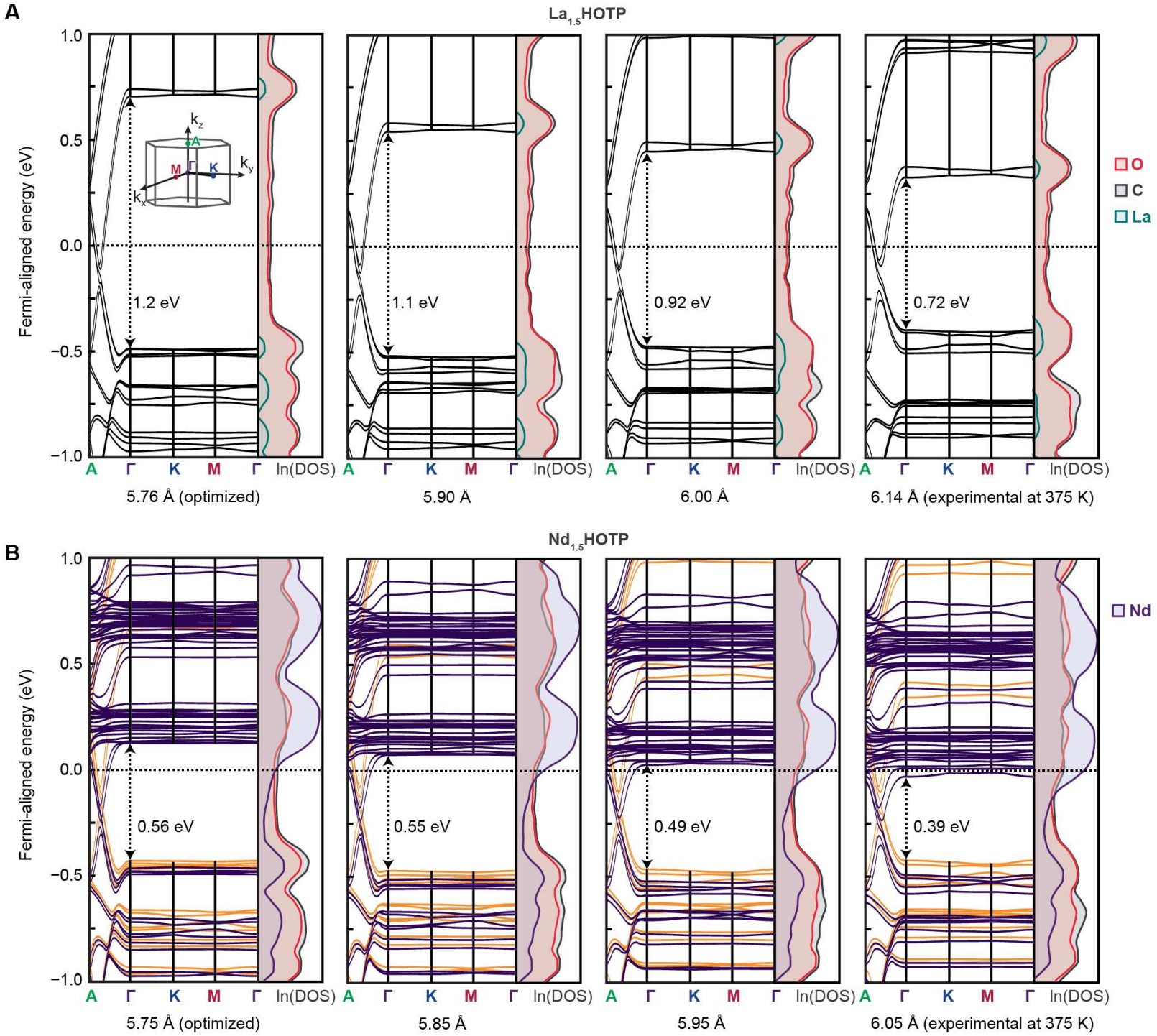

Fig. S10.

Electronic band structures of La1.5HOTP (panel A) and Nd1.5HOTP (panel B), calculated with different unit cell $c$ parameters between the optimized (leftmost), and experimental (rightmost). The overall features of the band structures are maintained through the unit cell parameter variation, limited mostly to narrowing of the band gap in the $\Gamma-\mathrm{K}-\mathrm{M}$ directions. 


\begin{tabular}{|c|c|c|}
\hline Compound & La1.5HOTP & Nd1.5HOTP \\
\hline Empirical formula & $\mathrm{C}_{36} \mathrm{H}_{12} \mathrm{O}_{19.731} \mathrm{La}_{3}$ & $\mathrm{C}_{18} \mathrm{H}_{6} \mathrm{O}_{9} \mathrm{Nd}_{1.5}$ \\
\hline Formula weight & 1176.9 & 582.59 \\
\hline Temperature/K & $375(2)$ & $375(2)$ \\
\hline Crystal system & monoclinic & monoclinic \\
\hline Space group & $P 2_{1} / n$ & $C 2 / c$ \\
\hline $\mathrm{a} / \AA \AA$ & $22.0779(3)$ & $38.018(7)$ \\
\hline $\mathrm{b} / \AA \AA$ & $38.303(2)$ & $21.936(4)$ \\
\hline $\mathrm{c} / \AA$ & $6.141(2)$ & $6.1056(10)$ \\
\hline$\alpha /^{\circ}$ & 90 & 90 \\
\hline$\beta /{ }^{\circ}$ & $89.582(2)$ & 90.104(7) \\
\hline$\gamma /{ }^{\circ}$ & 90 & 90 \\
\hline Volume $/ \AA^{3}$ & $5192.9(18)$ & $5091.8(16)$ \\
\hline $\mathrm{Z}$ & 4 & 8 \\
\hline$\rho_{\text {calc } g / \mathrm{cm}^{3}}$ & 1.5053 & 1.520 \\
\hline$\mu / \mathrm{mm}^{-1}$ & 2.485 & 3.072 \\
\hline $\mathrm{F}(000)$ & 2227.0 & 2208.0 \\
\hline Crystal size $/ \mathrm{mm}^{3}$ & $0.300 \times 0.040 \times 0.040$ & $0.400 \times 0.035 \times 0.035$ \\
\hline Radiation & $\operatorname{MoK} \alpha(\lambda=0.71073)$ & $\operatorname{MoK} \alpha(\lambda=0.71073)$ \\
\hline $2 \Theta$ range for data collection ${ }^{\circ}$ & 2.82 to 54.94 & 5.67 to 59.344 \\
\hline Index ranges & $\begin{array}{l}-28 \leq \mathrm{h} \leq 28,-49 \leq \mathrm{k} \leq \\
49,-7 \leq 1 \leq 7\end{array}$ & $\begin{array}{l}-52 \leq \mathrm{h} \leq 52,-30 \leq \mathrm{k} \leq \\
30,-8 \leq 1 \leq 7\end{array}$ \\
\hline Reflections collected & 151581 & 38024 \\
\hline Independent reflections & $\begin{array}{l}29094\left[\mathrm{R}_{\text {int }}=0.0478\right. \\
\left.\mathrm{R}_{\text {sigma }}=0.0420\right]\end{array}$ & $\begin{array}{l}7349\left[\mathrm{R}_{\text {int }}=0.0635,\right. \\
\left.\mathrm{R}_{\text {sigma }}=0.0445\right]\end{array}$ \\
\hline Data/restraints/parameters & $29094 / 12 / 567$ & 7349/474/293 \\
\hline Goodness-of-fit on $\mathrm{F}^{2}$ & 1.9597 & 1.052 \\
\hline Final R indexes $[\mathrm{I}>=2 \sigma(\mathrm{I})]$ & $\begin{array}{l}\mathrm{R}_{1}=0.0556, \quad \mathrm{wR}_{2}= \\
0.1222\end{array}$ & $\begin{array}{l}\mathrm{R}_{1}=0.0507, \quad \mathrm{wR}_{2}= \\
0.1456\end{array}$ \\
\hline Final $\mathrm{R}$ indexes [all data] & $\begin{array}{l}\mathrm{R}_{1}=0.0830, \quad \mathrm{wR}_{2}= \\
0.1275\end{array}$ & $\begin{array}{l}\mathrm{R}_{1}=0.0550, \quad \mathrm{wR}_{2}= \\
0.1496\end{array}$ \\
\hline Largest diff. peak/hole / e $\AA^{-3}$ & $1.38 /-1.43$ & $1.10 /-3.31$ \\
\hline
\end{tabular}

Table S1.

Selected details of structure collection and refinement. 


\begin{tabular}{|c|c|c|c|c|}
\hline Device number ${ }^{l}$ & Length, $\mu \mathrm{m}$ & $\begin{array}{l}\text { Diameter, } \\
\mu \mathrm{m}\end{array}$ & $\begin{array}{l}\text { Resistance, } \\
\Omega\end{array}$ & $\begin{array}{l}\text { Conductivity, } \\
\text { S/cm }\end{array}$ \\
\hline \multicolumn{5}{|c|}{ La $a_{1.5}$ HOTP batch 1} \\
\hline C1 (terminals 2-3) & 150 & 7.5 & 205 & 200 \\
\hline C1 (terminals 2-4) & 340 & 7.5 & 460 & 202 \\
\hline$C 2$ & 246 & 8.6 & 173 & 296 \\
\hline C4 (terminals 2-3) & 95 & 8.3 & 124 & 171 \\
\hline C4 (terminals 3-4) & 90 & 8.3 & 100 & 201 \\
\hline C4 (terminals 2-4) & 280 & 8.3 & 230.5 & 271 \\
\hline \multicolumn{5}{|c|}{ La $a_{1.5}$ HOTP batch 2} \\
\hline 14 & 36 & 12 & 19 & 203 \\
\hline 12 & 175 & 7.6 & 58.5 & 797 \\
\hline $2-B C$ & 233 & 8.6 & 54 & 898 \\
\hline $2-B D$ & 431 & 8.6 & 103.5 & 867 \\
\hline $15 v 2$ & 27.8 & 15.2 & 7.4 & 250 \\
\hline 17 & 281 & 9.5 & 77.6 & 618 \\
\hline \multicolumn{5}{|c|}{ La $a_{1.5}$ HOTP batch 3} \\
\hline 1 & 73 & 5 & 59 & 763 \\
\hline 2 & 28.5 & 14 & 7.6 & 295 \\
\hline \multicolumn{5}{|c|}{$N d_{1.5} H O T P$ batch 1} \\
\hline G11 & 70 & 54.6 & 1.2 & 301 \\
\hline G4 & 57.5 & 29 & 1.28 & 822 \\
\hline G10 & 77.5 & 47.7 & 1.3 & 403 \\
\hline G1 & 72.3 & 44 & 0.9 & 639 \\
\hline $\mathrm{G} 2$ & 41.4 & 30 & 1.6 & 443 \\
\hline \multicolumn{5}{|c|}{ Nd $d_{1.5}$ HOTP batch 2} \\
\hline $\mathrm{C} 4$ & 170 & 13.5 & 16.1 & 892 \\
\hline C6 & 62 & 18.3 & 13.1 & 218 \\
\hline $\mathrm{C} 7$ & 180 & 15.3 & 13.4 & 883 \\
\hline $\mathrm{Si}-1$ & 150 & 26 & 13 & 263 \\
\hline G2 & 122.5 & 12.9 & 20.6 & 550 \\
\hline \multicolumn{5}{|c|}{$N d_{1.5} H O T P$ batch 3} \\
\hline $\mathrm{C} 1$ & 168 & 7.3 & 53.8 & 902 \\
\hline $\mathrm{C} 5$ & 122.5 & 11.8 & 12.5 & 1084 \\
\hline C9 & 214 & 14.8 & 16.9 & 890 \\
\hline
\end{tabular}

\footnotetext{
${ }^{1}$ For some devices, five working contacts were placed and measurements of several configurations were possible. In those cases, the contact terminals were numbered sequentially 1-5 along the long dimension of the crystal. Voltage contacts used are listed in the table
} 
Table S2.

Single crystal conductivities for $\mathrm{Ln}_{1.5} \mathrm{HOTP}$. 


\begin{tabular}{|c|c|c|}
\hline Label & $\begin{array}{l}\text { Frequency }\left(\mathrm{cm}^{-1}\right) \text { at } \\
\text { Room Temperature }\end{array}$ & Mode \\
\hline A & $\begin{array}{c}1596,1592,1585,1580- \\
1560\end{array}$ & \\
\hline B & $1536,1518,1513-1512$ & \\
\hline $\mathrm{C}$ & $1459,1455,1449$ & \\
\hline D & $1430-1429$ & \\
\hline E & $1400-1384$ & \\
\hline $\mathrm{F}$ & $1370,1362,1338-1330$ & \\
\hline
\end{tabular}




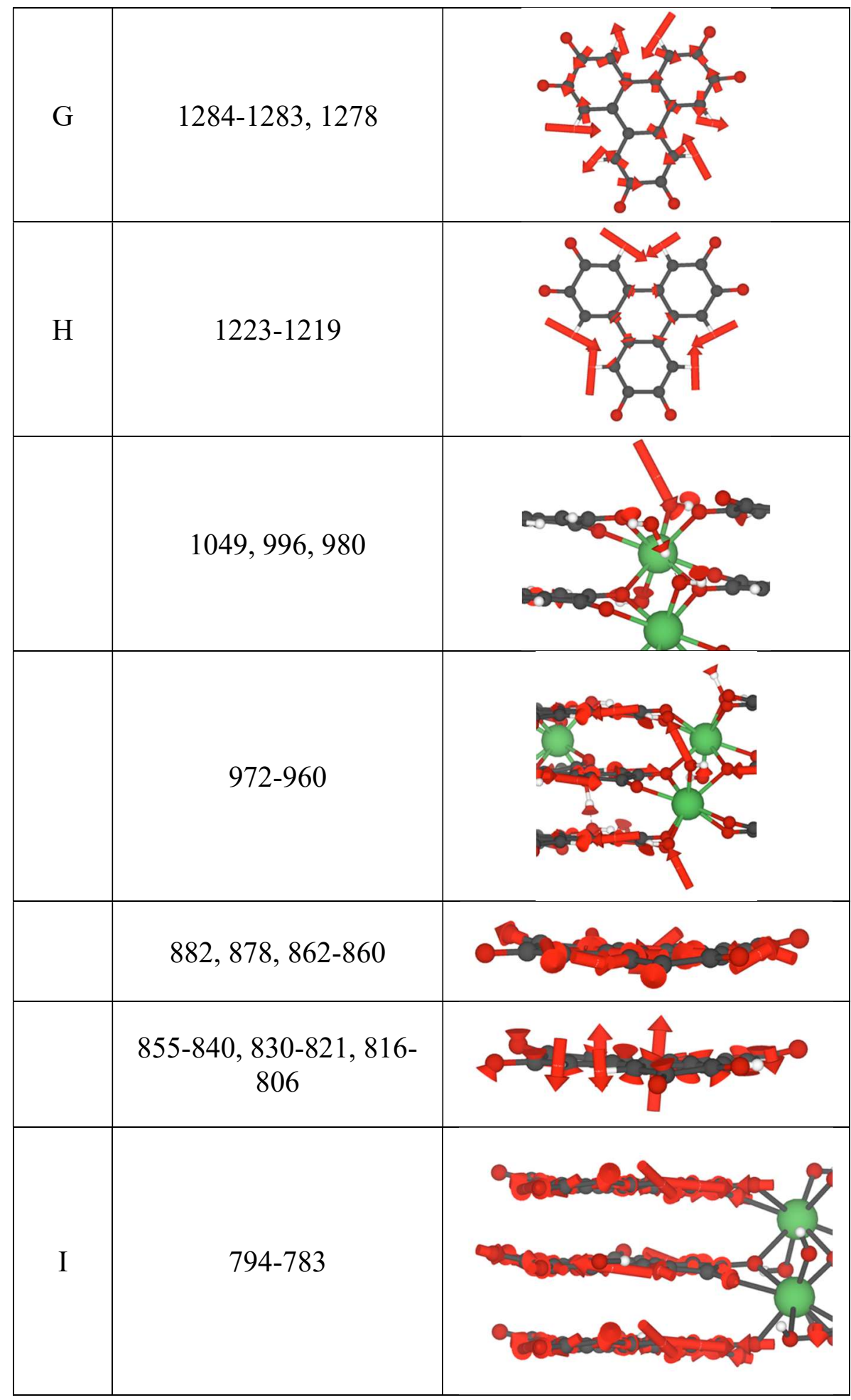




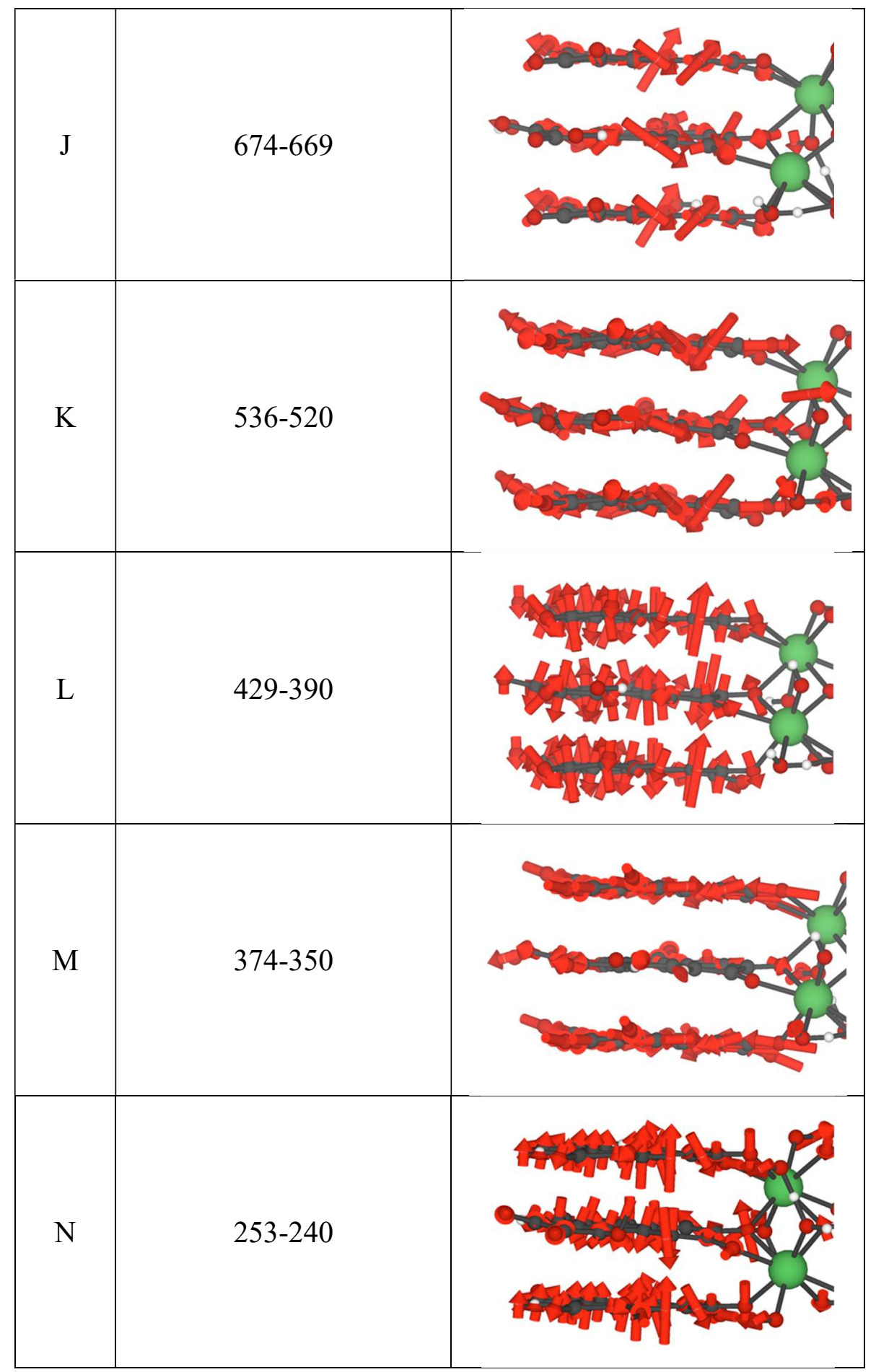




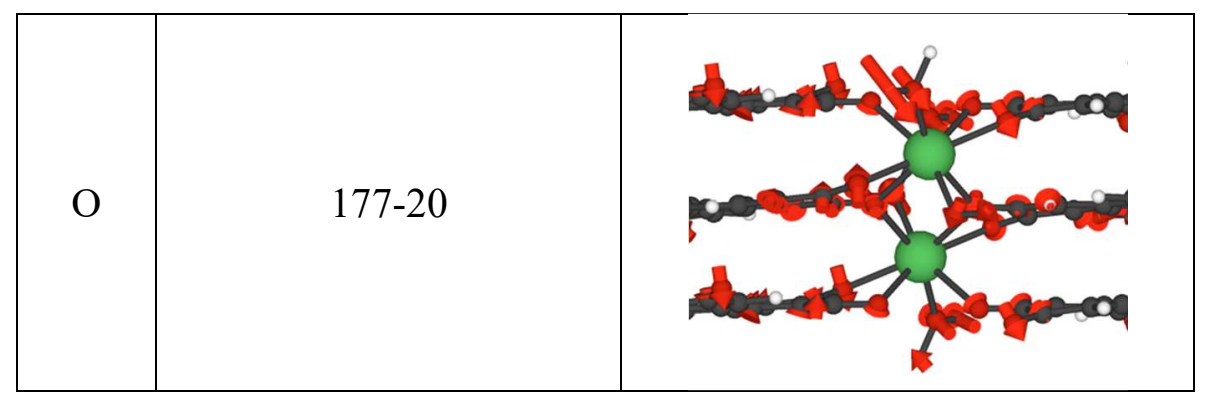

Table S3.

Visualization of vibrations for selected Raman modes in the DFT-simulated spectrum of La1.5HOTP. 


\section{References}

1. R. C. Borner, R. J. Bushby, A. N. Cammidge, Ferric chloride/methanol in the preparation of triphenylene-based discotic liquid crystals. Liq. Cryst. 33 (2006), pp. 1439-1448.

2. G. Skorupskii, M. Dincă, Electrical Conductivity in a Porous, Cubic Rare-Earth Catecholate. J. Am. Chem. Soc. 142, 6920-6924 (2020).

3. G. M. Sheldrick, A short history of SHELX. Acta Crystallogr. Sect. A Found. Crystallogr. 64 (2008), pp. 112-122.

4. V. Petříček, M. Dušek, L. Palatinus, Crystallographic Computing System JANA2006: General features. Zeitschrift für Krist. - Cryst. Mater. 229, 345-352 (2014).

5. G. M. Sheldrick, SHELXT - Integrated space-group and crystal-structure determination. Acta Crystallogr. Sect. A Found. Crystallogr. 71, 3-8 (2015).

6. $\quad$ L. Sun, S. S. Park, D. Sheberla, M. Dincă, Measuring and Reporting Electrical Conductivity in Metal-Organic Frameworks: $\mathrm{Cd}_{2}$ (TTFTB) as a Case Study. J. Am. Chem. Soc. 138, 14772-14782 (2016).

7. I. J. Bruno, J. C. Cole, P. R. Edgington, M. Kessler, C. F. Macrae, P. McCabe, J. Pearson, R. Taylor, IUCr, New software for searching the Cambridge Structural Database and visualizing crystal structures. Acta Crystallogr. Sect. B Struct. Sci. 58, 389-397 (2002).

8. G. Skorupskii, B. A. Trump, T. W. Kasel, C. M. Brown, C. H. Hendon, M. Dincă, Efficient and tunable one-dimensional charge transport in layered lanthanide metalorganic frameworks. Nat. Chem. 12, 131-136 (2020).

9. G. Kresse, J. Furthmüller, Efficient iterative schemes for ab initio total-energy calculations using a plane-wave basis set. Phys. Rev. B. 54, 11169-11186 (1996).

10. J. P. Perdew, A. Ruzsinszky, G. I. Csonka, O. A. Vydrov, G. E. Scuseria, L. A. Constantin, X. Zhou, K. Burke, Restoring the Density-Gradient Expansion for Exchange in Solids and Surfaces. Phys. Rev. Lett. 100, 136406 (2008).

11. F. Nilsson, R. Sakuma, F. Aryasetiawan, Ab initio calculations of the Hubbard U for the early lanthanides using the constrained random-phase approximation. Phys. Rev. B Condens. Matter Mater. Phys. 88, 125123 (2013). 